Original Research Paper

\title{
Hysteresis, Resonant Oscillations and Bifurcation Mode of a System Modeled by a Forced Modified Van der Pol-Duffing Oscillator
}

\author{
${ }^{1}$ Adjimon Vincent Monwanou, ${ }^{2}$ Laurent Amoussou Hinvi, \\ ${ }^{3}$ Clément Hodévèwan Miwadinou and ${ }^{1}$ Jean Bio Chabi Orou \\ ${ }^{1}$ Institut de Mathématiques et de Sciences Physiques de Dangbo, Bénin \\ ${ }^{2}$ Institut Universitaire de Technologie de Lokossa, Bénin \\ ${ }^{3}$ Ecole Normale Supérieure de Natitingou, Bénin
}

Article history

Received: 23-08-2017

Revised: 25-09-2017

Accepted: 05-10-2017

Corresponding Author:

Adjimon Vincent Monwanou Institut de Mathématiques et de Sciences Physiques de

Dangbo, Bénin

Email: movins2008@yahoo.fr

\begin{abstract}
Nonlinear oscillations and its applications in physics, chemistry, engineering, biophysics, communications are studied with some analytical, numerical and experimental methods. In the present paper, hysteresis, resonant oscillations and bifurcation mode of a system modeled by a forced modified Van der Pol-Duffing oscillator are considered. The plasma oscillations are considered and are described by a nonlinear differential equation. By using the harmonic balance technique and the multiple time scales methods, the amplitudes of the forced harmonic, superharmonic and subharmonic oscillatory states are obtained. Then, we derived admissible values of the amplitude of the external strength. Some bifurcation structures and transition to chaos of the model have been investigated. The model presented several dynamics motions which are influenced by nonlinear parameters. It can be concluded that the nonlinear parameters have a real impact on the dynamics of the model.
\end{abstract}

Keywords: Forced Modified Van der Pol-Duffing Oscillator, Harmonic Balance Technique, Resonant States, Hysteresis, Bifurcation

\section{Introduction}

The theory of oscillators has shown that many dynamics phenomena can be modeled by oscillators in engineering, biochemistry, biophysics, communications. Nonlinear oscillations and its applications in physics, chemistry, engineering, are studied with some analytical, numerical and experimental methods (Rajasekar et al., 1992). The most interesting nonlinear oscillators are self-excited and the study of their dynamics is often difficult. Duffing, Van der Pol and Rayleigh oscillators have been studied by many researchers (Miwadinou et al., 2016a; 2016b). Nowadays, much research has accomplished the composition of these oscillators. Multiresonance, chaotic behavior and its control, bifurcations, limit cycle stability, hysteresis and jump phenomena, analytic solutions, plasma oscillations, noise effect... are seriously analyzed (Miwadinou et al., 2015; 2014; Ainamon et al., 2014). Interests according to plasma oscillations are due to their potential applications (Dendy, 1993; Hsuan, 1968). It is important in some sectors such as biomedecine, automobiles, defence, aerospace, optics, solar energy, telecommunications, textiles, papers, polymers and waste management (Proud, 1991). For example, in particular, the nonlinear description of plasma oscillations is of interest as a result of its importance to the semiconductor industry (Mahaffey, 1976; Ostrikov, 2005). Experiments suggest that some plasma behavior is approximately described by anharmonic oscillations. Thus, it has been shown experimentally (Ostrikov and $\mathrm{Xu}, 2007$ ) and theoretically (Enjieu et al., 2007) that in plasma physics, the electron beam surfaces, the Tonks-Datter resonances of mercury vapor and low frequency ion sound waves, oscillations are described by the following anharmonic Equations (1) or (2) theoretically (Enjieu et al., 2008):

$$
\begin{aligned}
& \ddot{x}+\omega_{0}{ }^{2} x+\beta x^{2}+\gamma x^{3}=F \cos \Omega t \\
& \ddot{x}+\epsilon\left(1+x^{2}\right) \dot{x} \omega_{0}{ }^{2} x+\beta x^{2}+\gamma x^{3}=F \cos \Omega t
\end{aligned}
$$

In these equations $\omega_{0}$ and $\Omega$ are respectively the natural and external frequencies. $F$ stands for the amplitude of the external excitation while $\beta$; and $\gamma$ are the quadratic, cubic nonlinearities and damping parameters. 
Enjieu et al. (2008) studied with a rigorous theoretical consideration through a method based on the harmonic balance formalism the amplitude of the forced harmonic oscillations states. It is found the resonance states, admissible values of $F$ and bifurcation structures numerically. They found also the important effects of different parameters.

In the present paper, we focus our studies on the model and equation of motion, the resonant states, the chaotic behavior through hysteresis and bifurcation mode. Through these studies, we found the effects of parameters in general and in particular the effect of the hybrid quadratic parameter $\alpha$ which shown the difference between this equation and anharmonic equation obtained in (Enjieu et al., 2008).

The paper is structured as follows: Section 2 gives the equation of motion and amplitude of the forced harmonic oscillatory states of nonlinear dynamics of plasma oscillations. Section 3 investigates, using multiple time-scales method, the resonant states. Section 4 deals with bifurcation and chaotic behavior highlighted through numerical simulations. The conclusion is presented in the last section.

\section{Equation of Motion and Amplitude of the Forced Harmonic Oscillatory States}

The two-fluid model which treats the plasma as two inter penetrating conducting fluids has been source of growing interest for researchers for many years (Keen et al., 1972; 1971; Li and Matsuoka, 1996). Nowadays, it remains an interesting task due to its potential applications (Loverich and Shumlak, 2006; Bhattacharyya and Janaki, 2006; Shumlak and Loverich, 2003; Kanki et al., 2006; Arshad et al., 1999). The following nonlinear second order differential anharmonic equation governed the model:

$$
\ddot{x}+\epsilon\left(1+x^{2}\right) \dot{x}+x+\epsilon \alpha x \dot{x}+\beta x^{2}+\gamma x^{3}=F \cos \Omega t
$$

When the friction term vanishes $(\epsilon=0)$, then the equation reduces to forced modified Duffing oscillator equation (Enjieu et al., 2007) and $\epsilon \neq 0, \alpha=0$ leads to an anharmonic oscillator (Enjieu et al., 2008). Assuming that the fundamental component of the solution and the external excitation have the same period, the amplitude of harmonic oscillations can be tackled using the harmonic balance method (Hayashi, 1964). For this purpose, we express its solutions as:

$x=A \cos (\Omega-\psi) t+\xi$,

where, $A$ represents the amplitude of the oscillations and $\xi$ a constant. Inserting this solution Equation 4 in 3 and equating the constants and the coefficients of $\sin \omega t$ and $\cos \omega t$ and, we have:

$$
\begin{aligned}
& {\left[\left(1-\Omega^{2}\right) A+2 A \beta \xi+\gamma\left(\frac{3}{4} A^{3}+A \xi^{2}\right)\right]^{2}=} \\
& -\epsilon^{2}\left[A \Omega\left(1+\alpha \xi+\xi^{2}\right)+\frac{1}{4} \Omega A^{3}\right]^{2}+F^{2} \\
& \left(1+\frac{3}{2} \gamma A^{3}\right) \xi+\frac{1}{2} \beta A^{2}+\beta \xi^{2}+\gamma \xi^{3}=0
\end{aligned}
$$

If it is assumed that $|\xi| \ll|A|$, i.e., that shift in $x=0$ is small compared to the amplitude [3], then $\xi^{2}$ and $\xi^{3}$ terms in Eq. 6 can be neglected and one obtains:

$\xi=\frac{\beta A^{2}}{2+3 \gamma A^{2}}$

Substituting Equation 7 into 5, one obtains the following nonlinear algebraic equation:

$$
\begin{aligned}
& {\left[\frac{9}{4} \gamma^{2} A^{5}+\left(\frac{9}{2} \gamma-3 \gamma \Omega^{2}-2 \beta^{2}\right) A^{3}+2\left(1-\Omega^{2}\right)^{2} A\right]^{2}} \\
& +\epsilon^{2} \Omega^{2}\left[A\left(1+\frac{1}{4} A^{2}\right)\left(2+3 \gamma A^{2}\right)-\alpha \beta A^{3}\right]^{2} \\
& -F^{2}\left(2+3 \gamma A^{2}\right)^{2}
\end{aligned}
$$

This equation can be rewritten as:

$A^{10}+P_{1} A^{8}+P_{2} A^{6}+P_{3} A^{4}+P_{4} A^{2}-4 F=0$

With:

$$
\begin{aligned}
& P_{1}= \frac{\frac{9}{2} \gamma^{2}\left(\frac{9}{2} \gamma-3 \gamma \Omega^{2}-2 \beta^{2}\right)+\frac{9}{2} \epsilon^{2} \Omega^{2} \gamma^{2}+\frac{3}{8} \Omega^{2} \gamma-\frac{3}{2} \alpha \beta \Omega \epsilon^{2}}{\frac{9}{16\left(9 \gamma^{2}+\epsilon^{2} \Omega^{2}\right) \gamma^{2}}} \\
& P_{2}= \frac{9 \gamma^{2}\left(1-\Omega^{2}\right)+\left(\frac{9}{2} \gamma-3 \gamma \Omega^{2}-2 \beta^{2}\right)^{2}}{\frac{16\left(9 \gamma^{2}+\epsilon^{2} \Omega^{2}\right) \gamma^{2}}{4}} \\
&+\frac{-6 \alpha \beta \gamma \epsilon^{2} \Omega^{2} \gamma+\frac{\epsilon^{2} \Omega^{2}}{4}-\alpha \beta \epsilon^{2} \Omega^{2}+\epsilon^{2} \alpha^{2} \beta^{2} \Omega^{2}}{9} \\
& \frac{\left.9 \gamma^{2}+\epsilon^{2} \Omega^{2}\right) \gamma^{2}}{16\left(9 \gamma^{2}\right.}
\end{aligned}
$$




$$
\begin{aligned}
& P_{3}=\frac{4\left(1-\Omega^{2}\right)\left(\frac{9}{2} \gamma-3 \gamma \Omega^{2}-2 \beta^{2}\right)+6 \epsilon^{2} \Omega^{2}+6 \epsilon^{2} \Omega^{2} \gamma}{\frac{9}{16\left(9 \gamma^{2}+\epsilon^{2} \Omega^{2}\right) \gamma^{2}}} \\
& +\frac{\frac{-4 \alpha \beta \gamma \epsilon^{2} \Omega^{2}-9 \gamma^{2} F^{2}}{9}}{16\left(9 \gamma^{2}+\epsilon^{2} \Omega^{2}\right) \gamma^{2}} \\
& P_{3}=\frac{\frac{4\left(1-\Omega^{2}\right)^{2}-\gamma F^{2}}{9}}{16\left(9 \gamma^{2}+\epsilon^{2} \Omega^{2}\right) \gamma^{2}}
\end{aligned}
$$

The effects of the quadratic and cubic terms on the behavior of the amplitude of plasma oscillations are checked. The obtained results are reported in Fig. 1, 2 and 3. Hysteresis and jump phenomena are found. Hysteresis and jump phenomena known to be function of the cubic nonlinear coefficient and can also be triggered or quenched through the dissipative coefficient $\epsilon$ (Fig. 3) and as well as via the nonlinear quadratic parameter $\beta$ and $\alpha$ (Fig. 1 and 2). The behavior of the amplitude of plasma oscillations is investigated through Equation10 when the external frequency $\Omega$ varies. Thereby, the observed resonant state obtained for a set of parameters can be destroyed according to the value taken by the amplitude of the external force.

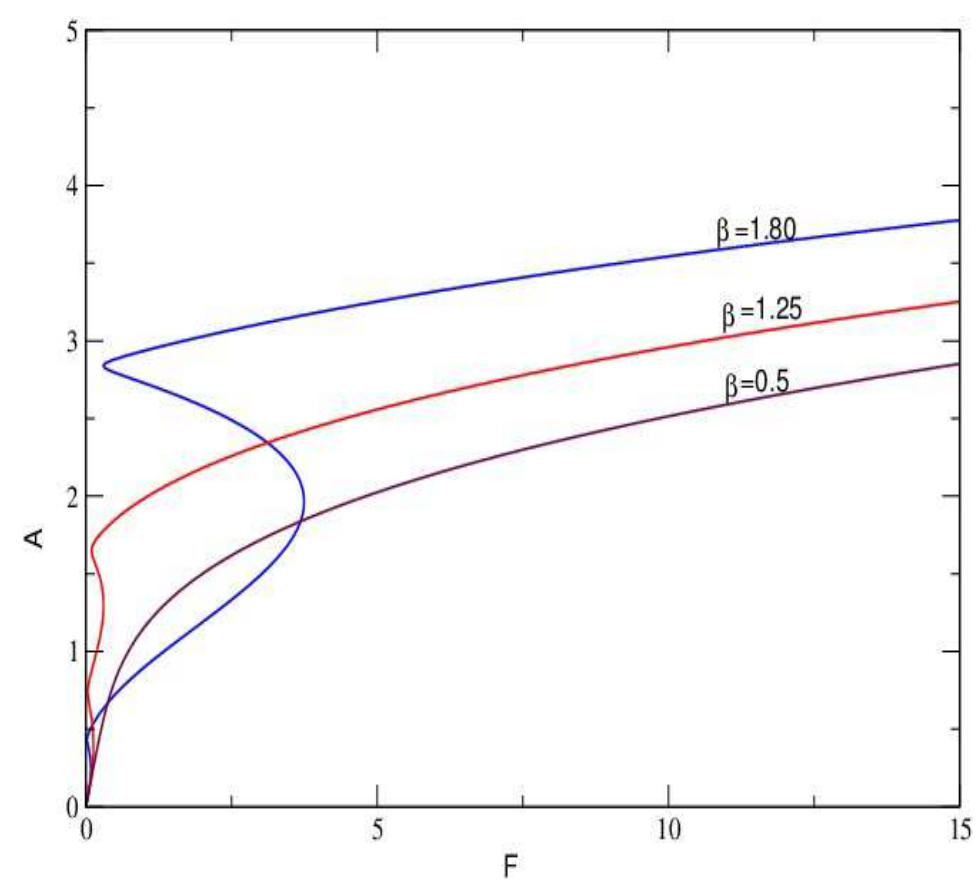

Fig. 1. Effects of $\beta$ on the amplitude-response curves with $\Omega=0.7, \gamma=0, \epsilon=0.05$ and $\alpha=0$

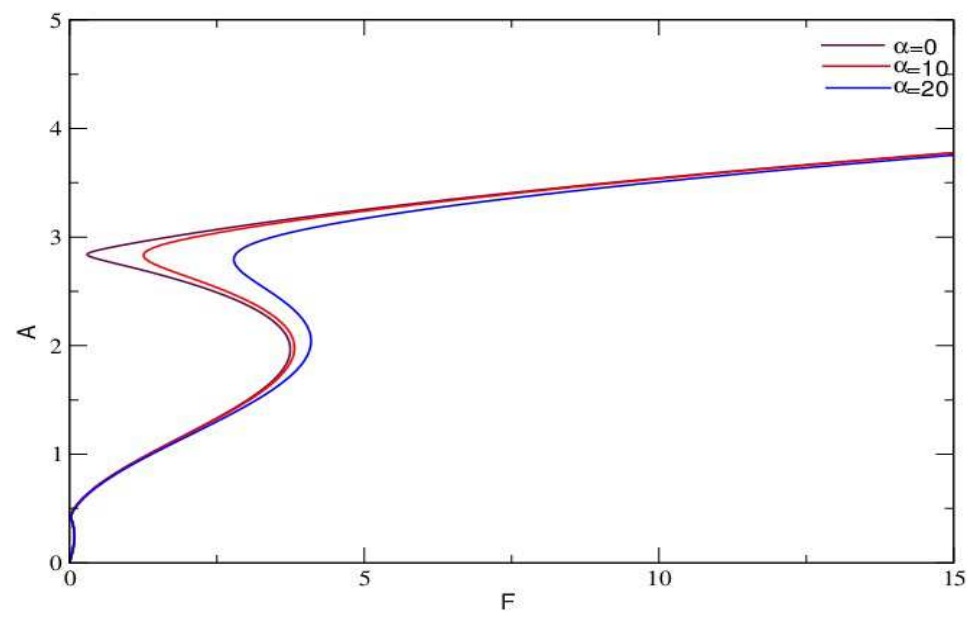

Fig. 2. Effects of $\alpha$ on the amplitude-response curves with $\Omega=0.7, \gamma=0, \epsilon=0.05$ and $\beta=1.85$ 


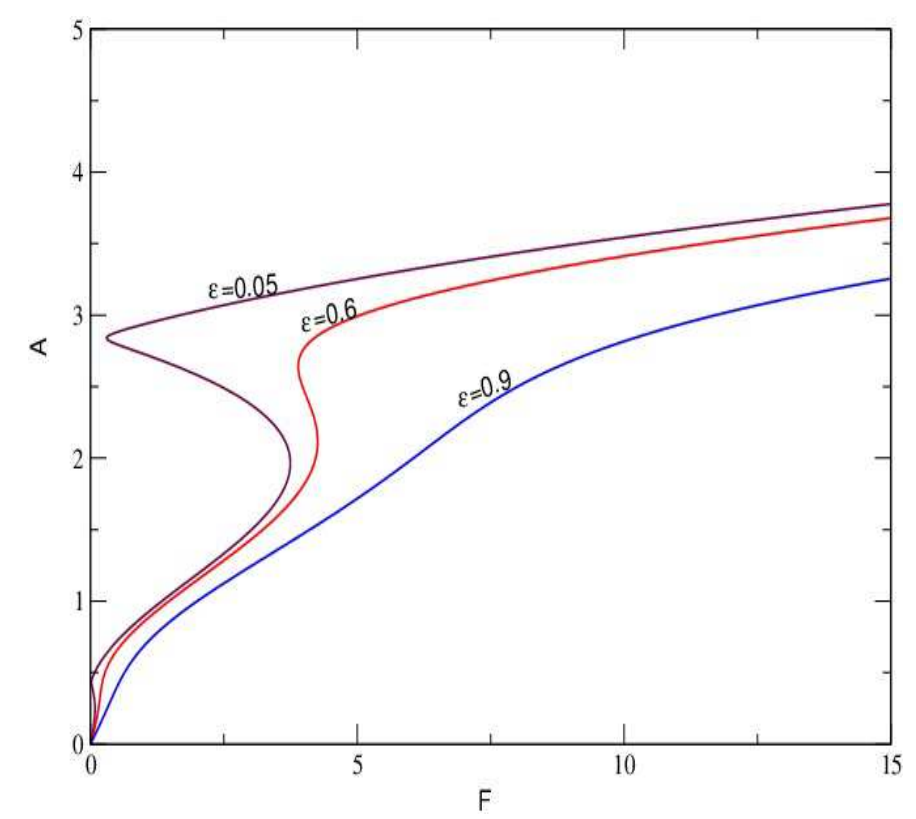

Fig. 3. Effects of $\epsilon$ on the amplitude-response curves with $\Omega=0.7, \gamma=0, \alpha=0$ and $\beta=1.85$

During the hysteresis and jump phenomena processes, for any value of the frequency $\Omega$ and the external force $F$ respectively, three different amplitudes of oscillations are obtained among which, two are stable and one is unstable.

\section{Resonant States}

The different resonances are investigated using the multiple time Scales Method (MSM).

In such situation, an approximate solution is generally sought as follows (Nayfeh, 1981):

$x(t, \epsilon)=x_{0}\left(T_{0}, T_{1}\right)+\epsilon x_{1}\left(T_{0}, T_{1}\right) \ldots$

With, $T_{n}=\epsilon^{n} t$

The derivatives operators can now be rewritten as follows:

$$
\begin{aligned}
& \left\{\frac{d}{d t}=D_{0}+\epsilon D_{1}\right. \\
& \left\{\frac{d^{2}}{d t^{2}}=D_{0}{ }^{2}+2 \epsilon D_{0} D_{1}\right.
\end{aligned}
$$

Where:

$$
D_{n}^{m}=\frac{\partial^{m}}{\partial T_{n}^{m}}
$$

\section{Primary Resonant State}

We put that $\mathrm{F} \approx \epsilon \mathrm{F}, \beta \approx \epsilon \beta$ and $\gamma \approx \epsilon \gamma$. The closeness between both internal and external frequencies is given by $\Omega=1+\epsilon \sigma$, where $\sigma$ is the detuning parameter. Inserting Equation 14 and 15 into 3, we obtain:

$$
\begin{aligned}
& \left(D_{0}^{2}+2 \epsilon D_{0} D_{1}\right)\left(x_{0}+\epsilon x_{1}\right) \\
& +\epsilon\left(1+\left(x_{0}+\epsilon x_{1}\right)^{2}\right)\left(D_{0}+\epsilon D_{1}\right)\left(x_{0}+\epsilon x_{1}\right)+\left(x_{0}+\epsilon x_{1}\right) \\
& +\epsilon \alpha\left(x_{0}+\epsilon x_{1}\right)\left(D_{0}+\epsilon D_{1}\right)\left(x_{0}+\epsilon x_{1}\right) \\
& +\beta\left(x_{0}+\epsilon x_{1}\right)^{2}+\gamma\left(x_{0}+\epsilon x_{1}\right)^{3}=\epsilon F \cos \Omega t \\
& D_{0}{ }^{2} x_{0}+x_{0}=0 \\
& D_{1}^{2} x_{1}+x_{1}=F \cos \Omega t-2 D_{1} D_{0} x_{0}-\left(1+x_{0}{ }^{2}\right)\left(D_{0} x_{0}\right) \\
& -\alpha x_{0} D_{0} x_{0}-\beta x_{0}{ }^{2}-\gamma x_{0}{ }^{3}
\end{aligned}
$$

The general solution of the first equation of Equation 18 is:

$$
x_{0}=A\left(T_{1}\right) \operatorname{Exp}\left(j T_{0}\right)+C C
$$

where, CC represents the complex conjugate of the previous terms. $A\left(T_{1}\right)$ is a complex function to be determined from solvability or secular conditions of Equation 19. Thus, substituting $x_{0}$ in Equation 19 leads us to the following secular criterion:

$\left[2 j A^{\prime}+j\left(1+|A|^{2}\right) A+3 \gamma|A|^{2} A-\frac{E}{2} e^{j \sigma T_{1}}\right]=0$

In polar coordinates, the solution of Equation 21 is:

$$
A\left(T_{1}\right)=\frac{1}{2} a\left(T_{1}\right) e^{j \theta\left(T_{1}\right)}+C C
$$

where, $\alpha$ and $\theta$ are real quantities and stand respectively for the amplitude and phase of oscillations. After injecting Equation 22 into 21, we separate real and 
imaginary terms and obtain the following coupled flow for the amplitude and phase:

$a^{\prime}=-\frac{a}{2}-\frac{a^{3}}{8}+\frac{F \sin \phi}{2}$

$\phi^{\prime} a=\alpha \sigma-\frac{3 \gamma a^{3}}{8}-\frac{F \cos \phi}{2}$

where, the prime denotes the derivative with respect to $T_{1}$ and $\phi=\sigma T_{1}-\theta\left(T_{1}\right)$. For the steady-state conditions $\left(\phi^{\prime}=a^{\prime}=0 \Leftrightarrow a_{0}=\phi_{0}=0\right)$, the following nonlinear algebraic equation is obtained:

$$
\frac{1+9 \gamma^{2}}{64} a_{0}^{6}+\frac{(1-6 \gamma \sigma)}{8} a_{0}^{4}+\frac{1+4 \sigma^{2}}{4} a_{0}^{2}-\frac{F^{2}}{4}=0
$$

where, $\alpha_{0}$ and $\phi_{0}$ are respectively the values of $\alpha$ and $\phi$ in the steady-state.

Equation 25 is the equation of primary resonance flow. Now, we study the stability of the process, we assume that each equilibrium state is submitted to a small perturbation as follow:

$$
\begin{aligned}
& \alpha=\alpha_{0}+\alpha_{1} \\
& \phi=\phi_{0}+\phi_{1}
\end{aligned}
$$

where, $a_{1}$ and $\phi_{1}$ are slight variations. Inserting Equation 26 into 27 and canceling nonlinear terms enable us to obtain:

$$
\begin{aligned}
& a_{1}^{\prime}=-\frac{1}{2}\left(1+\frac{3 a_{0}^{2}}{4}\right) \alpha_{1}-\alpha_{0}\left(\sigma-\frac{3 a_{0}^{2} \gamma}{8}\right) \phi_{1} \\
& \phi_{1}^{\prime}=\frac{a_{1}}{a_{0}}\left(\sigma-\frac{9 a_{0}^{2} \gamma}{8}\right)-\frac{1}{2}\left(1+\frac{a_{0}^{2}}{4}\right) \phi_{1}
\end{aligned}
$$

The stability process depends on the sign of eigenvalues $\Gamma$ of the Equation 28 and 29 which are given through the following characteristic equation:

$$
\Gamma^{2}+2 Q \Gamma+R=0
$$

Where:

$$
\left\{\begin{array}{c}
Q=\frac{1}{2}\left(2+p_{0}{ }^{2}\right) \\
R=\frac{1}{4}\left(1+\frac{3 a_{0}^{2}}{4}\right)\left(1+\frac{a_{0}^{2}}{4}\right)-\left(\sigma-\frac{3 a_{0}^{2} \gamma}{8}\right)\left(\sigma-\frac{9 a_{0}^{2} \gamma}{8}\right)
\end{array}\right.
$$

Since $Q>0$, the steady-state solutions are stable if $R>0$ and unstable otherwise. Figure 4 displays the amplitude response curves obtained from Equation 25 for different values of the parameter and one can notice that as it increases, the model goes from resonance to a hysteresis state.

\section{Superharmonic and Subharmonic Oscillations}

When the amplitude of the sinusoidal external force is large, other type of oscillations can be displayed by the model, namely the superharmonic and the subharmonic oscillatory states. It is now assumed that $F \approx \epsilon^{0} \bar{F}$ and therefore, one obtains the following equations at different order of $\epsilon$. In order $\epsilon^{0}$ :

$D_{0}^{2} x_{0}+x_{0}=F \cos \Omega t$,

$D_{1}^{2} x_{1}+x_{1}=-2 D_{1} D_{0} x_{0}-\left(1+x_{0}^{2}\right)\left(D_{0} x_{0}\right)$

$-\alpha x_{0} D_{0} x_{0}-\beta x_{0}^{2}-\gamma x_{0}^{3}$

The general solution of Equation 32 is:

$x_{0}=A\left(T_{1}\right) e^{j T_{0}}+\Lambda e^{j \Omega T_{0}}$ with $\Lambda=\frac{F}{2\left(1-\Omega^{2}\right)}$

Substituting the general solution $x_{0}$ into Equation 32, after some algebraic manipulations, we obtain:

$$
\begin{aligned}
& D_{1}^{2} x_{1}+x_{1}=\left[-2 j A^{\prime}-j\left(\left(1+|A|^{2}+2 \Lambda^{2}\right)+3 \gamma|A|^{2}+6 \gamma \Lambda^{2}\right)\right] e^{j T_{0}}+ \\
& -\left[j \Omega\left(1+\Lambda^{2}+2|A|^{2}\right)+3 \gamma|\Lambda|^{2}+6 \gamma|A|^{2}\right] \Lambda e^{j \Omega T_{0}}-(j+\gamma) A^{3} e^{3 j T_{0}} \\
& -[j(1+2 \Omega)+3 \gamma] A \Lambda^{2} e^{j(1+2 \Omega) T_{0}}-[j(2+\Omega)+3 \gamma] A^{2} \Lambda e^{j(2+\Omega) T_{0}} \\
& -[j(2-\Omega)+3 \gamma] A^{2} \Lambda e^{j(2-\Omega) T_{0}}-(j \Omega+\gamma) \Lambda^{3} e^{3 j \Omega T_{0}} \\
& -[j(1-2 \Omega)+3 \gamma] A \Lambda^{2} e^{j(1-2 \Omega) T_{0}}- \\
& (j \alpha+\beta) A^{2} e^{2 j T_{0}}-(j \alpha \Omega+\beta) \Lambda^{2} e^{2 j T_{0}}- \\
& {[j \alpha(1+\Omega)+2 \beta] A \Lambda e^{j(1+\Omega) T_{0}}-[j \alpha(1-\Omega)+2 \beta] A \Lambda e^{j(1-\Omega) T_{0}}} \\
& -2 \beta\left(|A|^{2}+\Lambda^{2}\right)+j \alpha\left(|A|^{2}+\Omega \Lambda^{2}\right)+C C
\end{aligned}
$$

From Equation 34, it comes that Superharmonic and subharmonic states can be found from the quadratic and cubic nonlinearities. The cases of superhamonic oscillations we consider are $3 \Omega=1+\epsilon \sigma$ and $2 \Omega=1+\epsilon \sigma$ while the subharmonic oscillations to be treated are $\Omega=3+\epsilon \sigma$ and $\Omega=2+\epsilon \sigma$.

For the first superhamonic states $3 \Omega=1+\epsilon \sigma$, equating resonant terms at 0 from Equation 34, we obtain:

$$
\left[-2 j A^{\prime}+j\left[\begin{array}{l}
\left.1+|A|^{2}+2 \Lambda^{2}\right] A+3 \gamma|A|^{2} A-6 \gamma \Lambda^{2} A \\
-(j \Omega+\gamma) \Lambda^{3} e^{j \epsilon \sigma T_{0}}
\end{array}\right]=0\right.
$$

Using Equation 22 and after some algebraic manipulations, we rewrite Equation 35 as follows:

$$
\begin{aligned}
& a^{\prime}=-\frac{a}{2}+\frac{a^{3}}{8}-a \Lambda^{2}-\Lambda^{3}(\Omega \cos \phi+\gamma \sin \phi) \\
& a \phi^{\prime}=a \sigma-\frac{3 \gamma a^{3}}{8}-3 \gamma \Lambda^{2} a+\Lambda^{3}(\gamma \cos \phi-\Omega \sin \phi)
\end{aligned}
$$


The amplitude of oscillations of this superharmonic states is governed by the following nonlinear algebraic equation:

$$
\left(\frac{a_{0}}{2}+\frac{a_{0}{ }^{3}}{8}+a_{0} \Lambda^{2}\right)^{2}+\left(a_{0} \sigma-\frac{3 \gamma a_{0}{ }^{3}}{8}-3 \gamma a_{0} \Lambda^{2}\right)^{2}-\left(\gamma^{2}+\Omega^{2}\right) \Lambda^{2}
$$

After some algebraic manipulations, Equation 38 can be rewritten as follows:

$$
\begin{aligned}
& \frac{1+9 \gamma^{2}}{64} a_{0}^{6}+\frac{1}{4}\left[\frac{1}{2}+\Lambda^{2}-3 \gamma\left(\sigma-3 \gamma \Lambda^{2}\right)\right] a_{0}^{4} \\
& +\left[\left(\frac{1}{2}+\Lambda^{2}\right)^{2} 6\left(\sigma-3 \gamma \Lambda^{2}\right)^{2}\right] a_{0}^{2}+\left(\gamma^{2}+\Omega^{2}\right) \Lambda^{6}=0
\end{aligned}
$$

And they are stable if:

$$
\begin{aligned}
& \frac{1}{4}\left(1+2 \Lambda^{2}+\frac{3}{4} \alpha_{0}^{2}\right)\left(1+2 \Lambda^{2}+\frac{1}{4} \alpha_{0}^{2}\right)+\left(\begin{array}{c}
\sigma-\frac{9}{8} \gamma \alpha_{0}^{2} \\
-3 \gamma \Lambda^{2}
\end{array}\right) \\
& \left(\sigma-\frac{3}{8} \gamma \alpha_{0}-3 \gamma \Lambda^{2}\right)>0
\end{aligned}
$$

Figure 5 presents the frequency response curves of the superharmonic resonance as a function of $\sigma$ for different values of the external force. As the intensity of the external force increases, the resonance behavior observed is destroyed. The effects of the parameter on such superharmonic oscillations are also investigated and results are reported in Fig.6, showing the appearance of the hysteresis phenomenon when the nonlinear cubic parameter is increasing.
On the other hand, for the second superharmonic states $2 \Omega=1+\epsilon \sigma$ by inserting this condition into Equation 34 and equating the secular terms to 0 , we obtain:

$\left[-2 j A^{\prime}-j\left[\begin{array}{l}\left.1+|A|^{2}+2 \Lambda^{2}\right] A+3 \gamma|A|^{2} A+6 \gamma \Lambda^{2} A- \\ (j \alpha \Omega+\beta) \Lambda^{2} e^{j \epsilon \sigma T_{0}}\end{array}\right]=0\right.$

After same algebraic manipulations as in the first superhamonic resonance, we obtain:

$$
\begin{aligned}
& \frac{1+9 \gamma^{2}}{64} \mathrm{a}_{0}^{6}+\frac{1}{4}\left[\frac{1}{2}+\Lambda^{2}-3 \gamma\left(\sigma-3 \gamma \Lambda^{2}\right)\right] \mathrm{a}_{0}^{4} \\
& -\left[\left(\frac{1}{2}+\Lambda^{2}\right)^{2}-\left(\sigma-3 \gamma \Lambda^{2}\right)^{2}\right] \mathrm{a}_{0}^{2}+\left(\alpha^{2} \Omega^{2}+\beta^{2}\right) \Lambda^{4}=0
\end{aligned}
$$

and the stability criterion is the one defined through inequality Equation 40. In this cases also, the influence of the quadratic parameter on such oscillations has been checked checked (Fig. 7 and 8). In such a state, we noticed that the peak values of this superharmonic resonance are increased progressively when $\beta$ or $\alpha$ are increased.

The first subharmonic oscillations $\Omega=3+\epsilon \sigma$ are fund. Inserting this condition into Equation 35 and equating the secular terms to 0 , we obtain:

$$
\left[-2 j A^{\prime}-j\left[\begin{array}{l}
\left.1+|A|^{2}+2 \Lambda^{2}\right] A+3 \gamma|A|^{2} A+6 \gamma \Lambda^{2} A \\
-(j(2-\Omega)+3 \gamma) \Lambda \bar{A}^{2} e^{j \epsilon \sigma T_{0}}
\end{array}\right]\right.
$$

With Equation 22, 43 gives after some algebraic manipulations and separating real and imaginary terms:

$a^{\prime}=-\frac{a}{2}-\frac{a^{3}}{8}-a \Lambda^{2}-\frac{1}{4} \Lambda a^{2}((2-\Omega) \cos \phi+3 \gamma \sin \phi)$

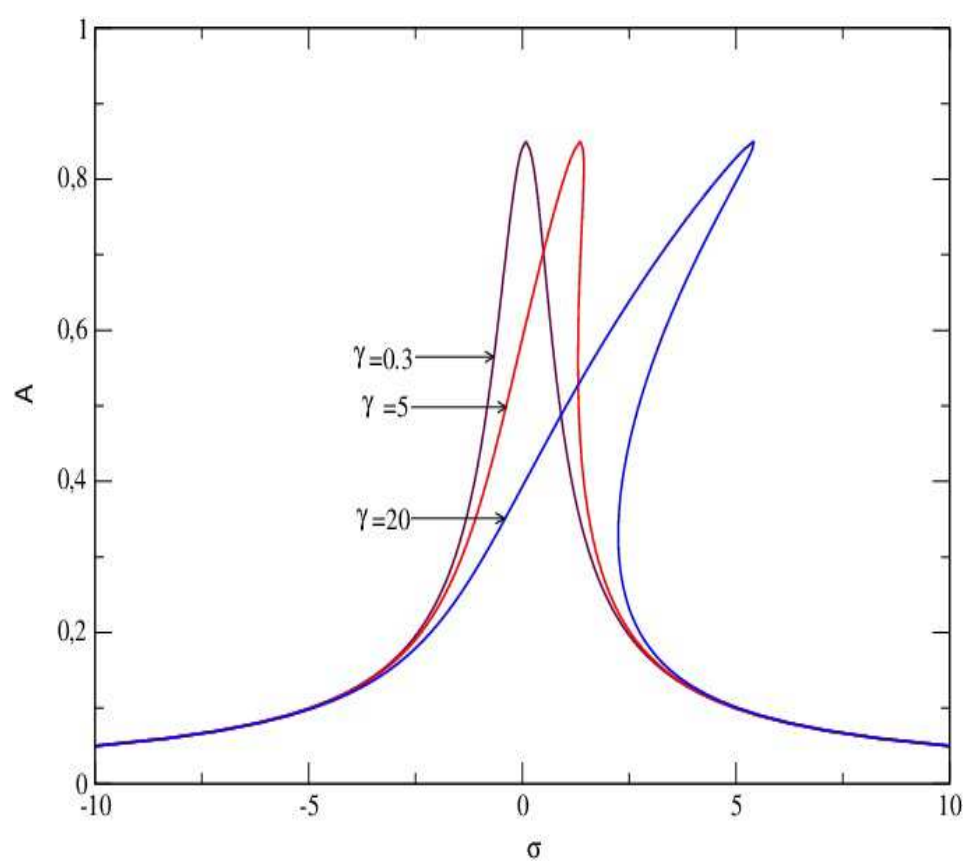

Fig. 4. Effects of on the frequency-response curves of the primary resonance with the parameters for $F=1.0$ 


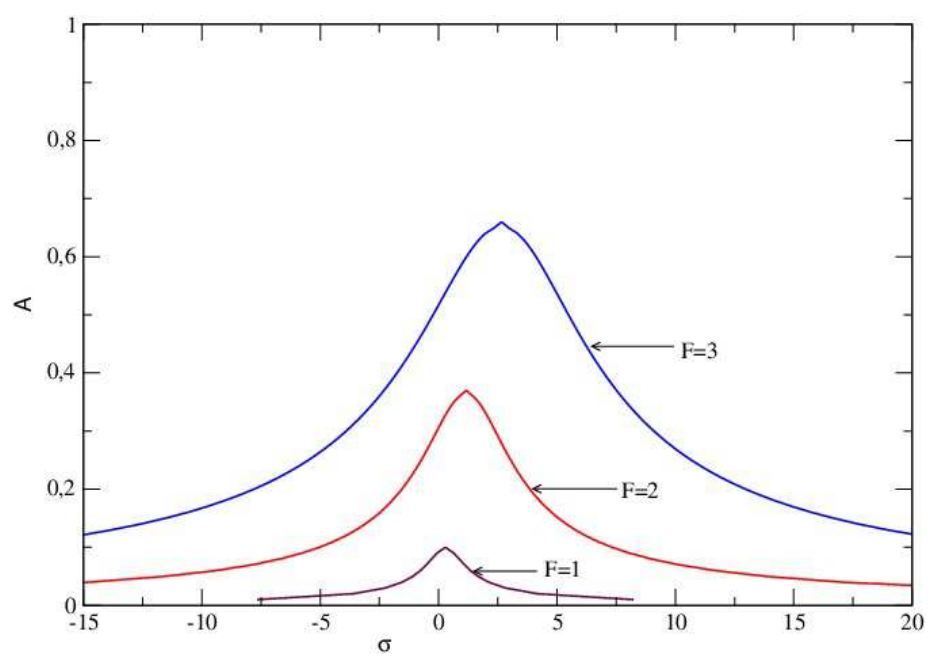

Fig. 5. Effects of $\mathrm{F}$ on the frequency-response curves of the order-3 superharmonic resonance with $\gamma=0.3$

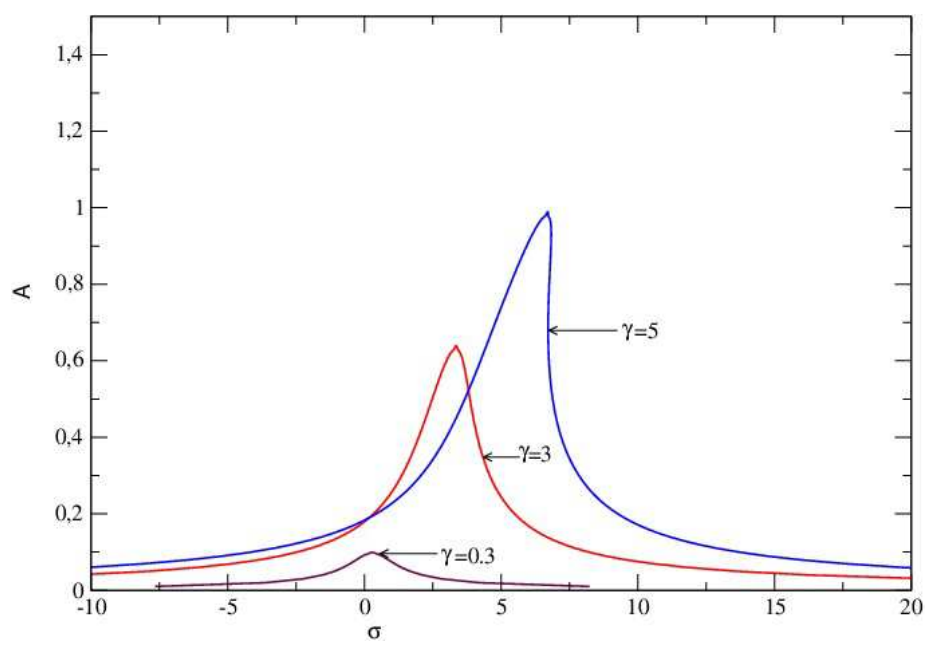

Fig. 6. Effects of $\gamma$ on the frequency-response curves of the order- 3 superharmonic resonance with $F=1$

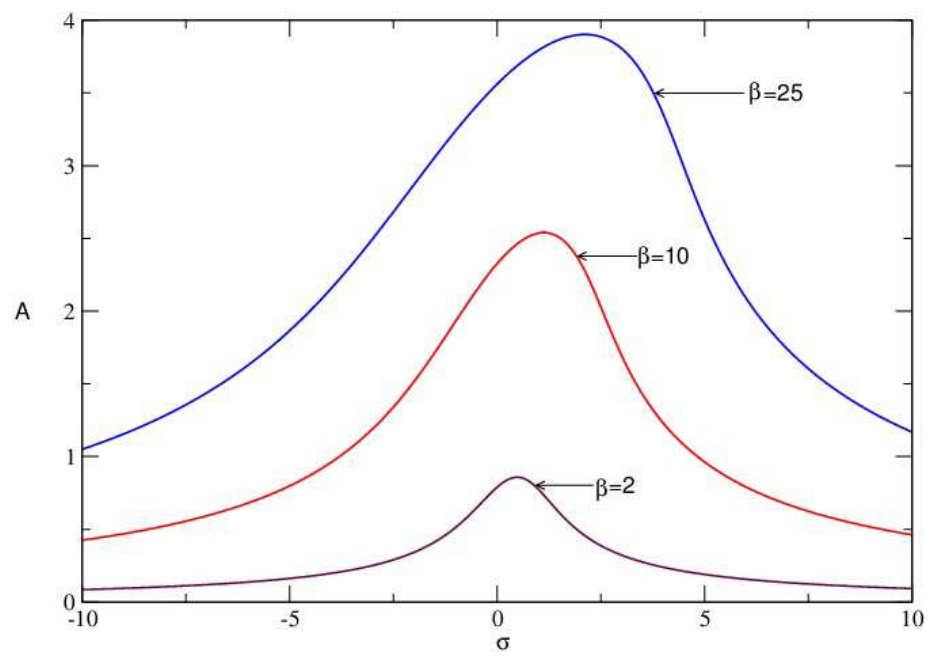

Fig. 7. Effects of $\beta$ on the frequency-response curves of the order- 2 superharmonic resonance with $F=1$ 


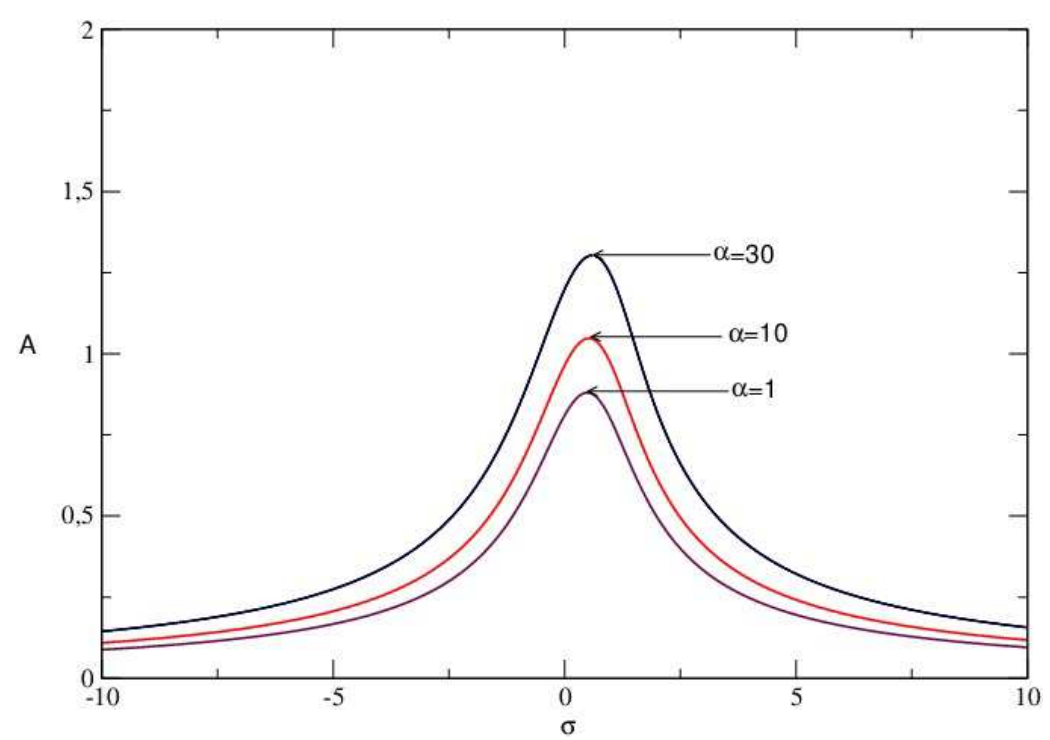

Fig. 8. Effects of on the frequency-response curves of the order-2 superharmonic resonance with $F=1$ and $\beta=2$

$$
a \phi^{\prime}=a \sigma-\frac{3 \gamma a^{3}}{8}-3 \gamma \Lambda^{2} a+\frac{1}{4} \Lambda a^{2}
$$

$(3 \gamma \cos \phi-(2-\Omega) \sin \phi)$

$$
\begin{aligned}
& \left(\frac{a_{0}}{2}+\frac{a_{0}{ }^{3}}{8}+a_{0} \Lambda^{2}\right)^{2}+\left(\frac{1}{3} a_{0} \sigma-\frac{3 \gamma a_{0}{ }^{3}}{8}-3 \gamma a_{0} \Lambda^{2}\right)^{2} \\
& -\frac{1}{16} \Lambda^{2} a_{0}{ }^{4}\left(9 \gamma^{2}+(2-\Omega)^{2}\right)=0
\end{aligned}
$$

Finally the amplitude of subharmonic oscillatory states is given by the following nonlinear algebraic equation:

$$
\begin{aligned}
& \frac{1+9 \gamma^{2}}{64} a_{0}^{4} \\
& +\frac{1}{4}\left[\frac{1}{2}+\Lambda^{2}-3 \gamma\left(\frac{\sigma}{3}-3 \gamma \Lambda^{2}\right)\right] a_{0}^{2} \\
& \left.-\frac{1}{16} \Lambda^{2}\left(9 \gamma^{2}+(2-\Omega)^{2}\right)\right] \\
& -\left[\left(\frac{1}{2}+\Lambda^{2}\right)^{2}+\left(\frac{\sigma}{3}-3 \gamma \Lambda^{2}\right)^{2}\right]=0
\end{aligned}
$$

and they are stable if:

$$
\begin{aligned}
& \left(\frac{1}{2}+\Lambda^{2}+\frac{1}{4} a_{0}^{2}\right)\left(\frac{1}{2}+\Lambda^{2}+\frac{1}{8} a_{0}^{2}\right) \\
& -\left(\frac{\sigma}{9}-\frac{a_{0}^{2}}{8}-\gamma \Lambda^{2}\right)\left(\sigma+\frac{9}{8} \gamma a_{0}^{2}-9 \gamma \Lambda^{2}\right)<0
\end{aligned}
$$

$$
\left[\begin{array}{l}
\left.\left(1+\left.A\right|^{2}+2 \Lambda^{2}\right)+3 \gamma|A|^{2}+6 \gamma \Lambda^{2}\right] A \\
-(j(1-\Omega) \alpha+2 \beta) \Lambda \bar{A} e^{j \epsilon \sigma T_{0}}
\end{array}\right]
$$

We obtain that the second subharmonic oscillatory states motions are governed by the equation:

$$
\begin{aligned}
& \frac{1+9 \gamma^{2}}{64} a_{0}^{6} \\
& +\left[\frac{1}{8}+\frac{\Lambda^{2}}{4}-\frac{3 \gamma}{4}\left(\frac{\sigma}{2}-3 \gamma \Lambda^{2}\right)\right] a_{0}^{2} \\
& +\left[\left(\frac{1}{16} \Lambda^{2}\left(9 \gamma^{2}+(2-\Omega)^{2}\right)\right]\right. \\
& -\left[\left(\frac{1}{2}+\Lambda^{2}\right)^{2}+\left(\frac{\sigma}{2}-3 \gamma \Lambda^{2}\right)^{2}\right] \\
& -\left(1-\Omega^{2}\right) \alpha^{2} \Lambda^{2} \beta^{2}-4 \Lambda^{2} \beta^{2}=0
\end{aligned}
$$

They are stable if the following criterion is fulfilled:

$$
\left(\frac{1}{2}+2 \Lambda^{2}+\frac{1}{4} a_{0}^{2}\right) a_{0}^{2}+6\left[\left(\frac{\sigma}{2}-3 \gamma \Lambda^{2}\right) a_{0}^{2}-\frac{3}{8} \gamma a_{0}^{2}\right]>0
$$

Now, we consider the second subharmonic oscillatory states $\Omega=2+\epsilon \sigma$. This condition implies althrough Equation 34 the secular term which equating 0 give:
The frequency response curves of both types of subharmonic oscillations are plotted in Figs. 9 and 10 and the regions where such behaviors occur are obtained. From these pictures, it comes that the range of frequency where a response can be obtained is more important in the first subharmonic state than in the other cases. 


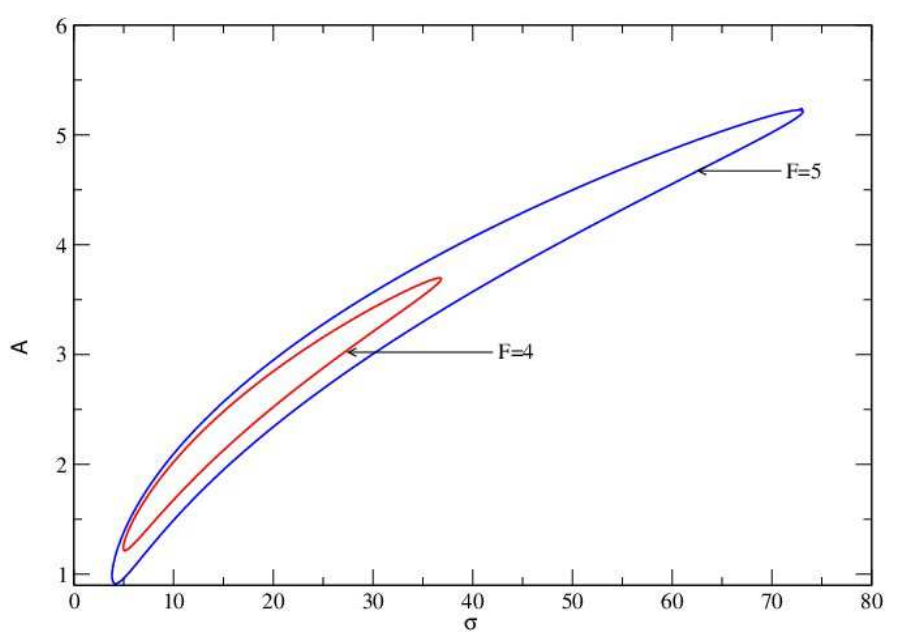

Fig. 9. Effects of $F$ on the frequency-response curves of the order-3 subharmonic resonance with $\gamma=2.30$
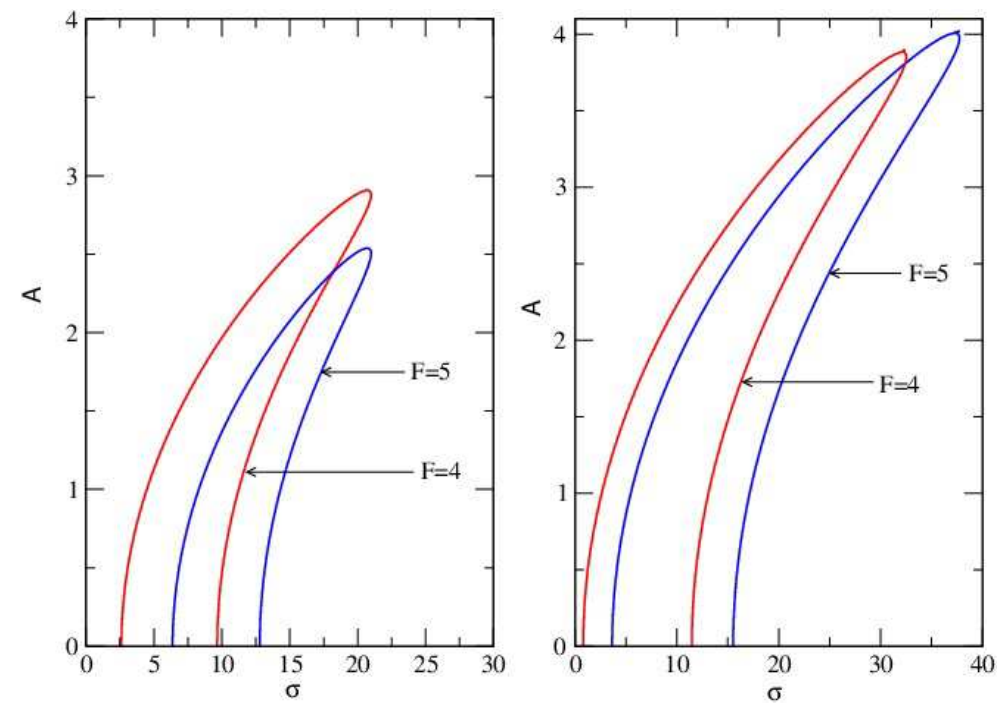

Fig. 10. Effects of $F$ on the frequency-response curves of the first subharmonic resonance with $\gamma=2.30 ; \beta=1 ;$ left $\alpha=0$ and right $\alpha=1$

\section{Bifurcation and Chaotic Behavior}

The aim of this section is to find some bifurcation structures in the nonlinear dynamics of plasma oscillations described by Equation 3 for resonant states since they are of interest in this system. For this purpose, we numerically solve this equation using the fourth-order Runge Kutta algorithm (Piskunov, 1980). We plot the resulting bifurcation diagrams and the variation of the corresponding largest Lyapunov:

$$
\operatorname{lyn}=\lim _{t \rightarrow \infty} \frac{\ln \sqrt{d x^{2}+d \dot{x}^{2}}}{t}
$$

where, $d x$ and $d \dot{x}$ are respectively the variations of $x$ and $\dot{x}$. Initial condition that we used in the simulations of this section is $\left(x_{0}, \dot{x}_{0}\right)=(1,1)$. For the set of parameters $\beta=3.05, \gamma=1.5, \epsilon=0.02, \Omega=1$ (lift), with $\alpha=1$ (right), the bifurcation and Lyapunov exponents diagrams for primary, superharmonic and subharmonic resonances are plotted in Fig. 11. The same simulations are found in Fig. 12 and 13 respectively with $\beta=6$ and $\beta=1$, $80 ; \epsilon=0,06$. From the bifurcation diagrams, various types of motions are displayed. It is found that the model can switch from periodic to quasi-periodic oscillations or chaotic motions as showing by Fig.11. In order to illustrate such situations, we have represented the various phase portraits using the parameters of the bifurcation diagram for which periodic, quasi-periodic oscillations and chaotic motions are observed in Fig. 14 with effect of parameter $\alpha$ in Fig. 15. These observations prove that the model is highly connected to the initial conditions. 

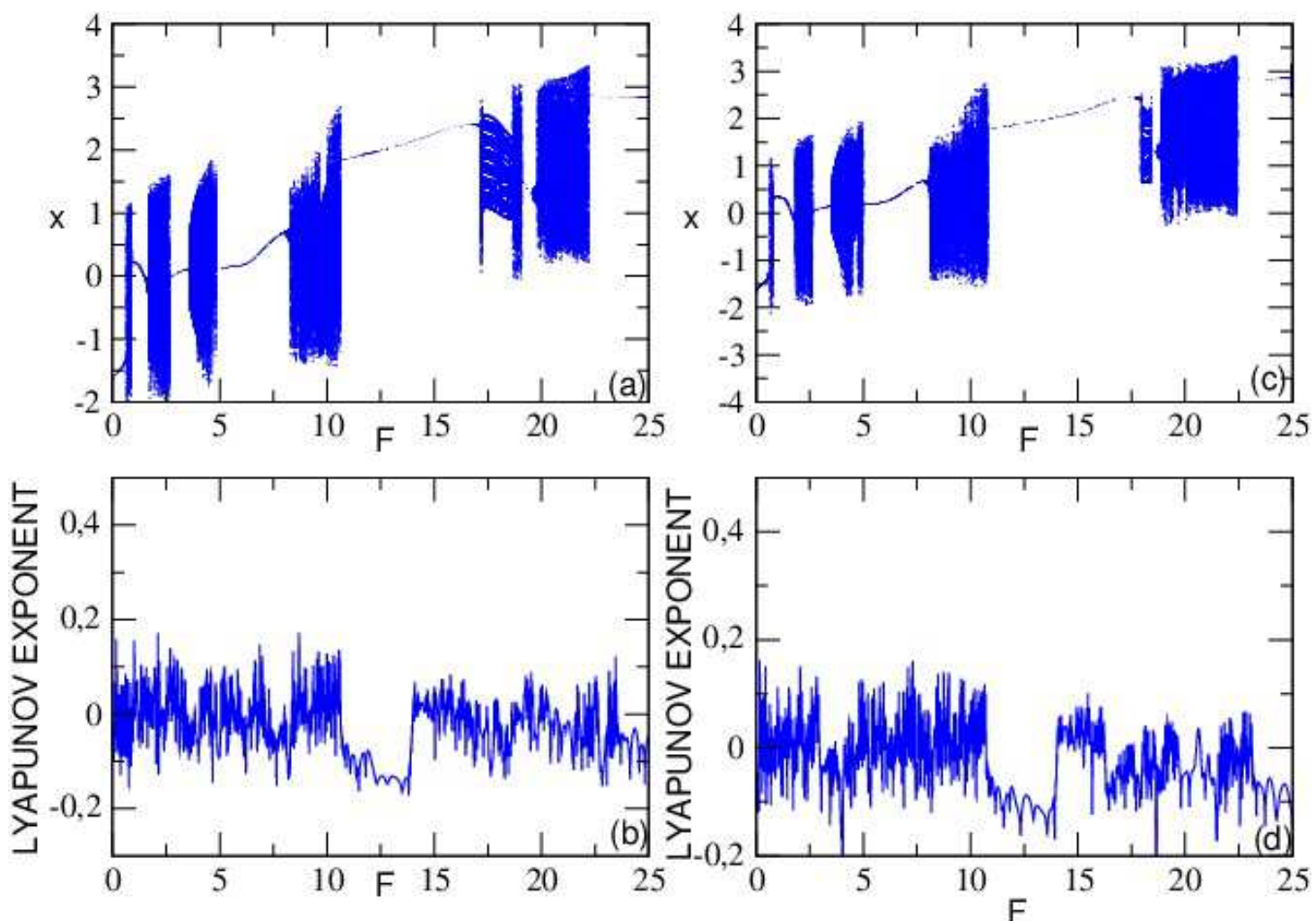

Fig. 11. Bifurcation diagram (upper frame) and Lyapunov exponent (lower frame) versus the amplitude $F$ with the parameters $\beta=3.05 ; \gamma=1.5 ; \Omega=1 ; \epsilon=0.02 ; \alpha=0$ (left) and $\alpha=1$ (right)
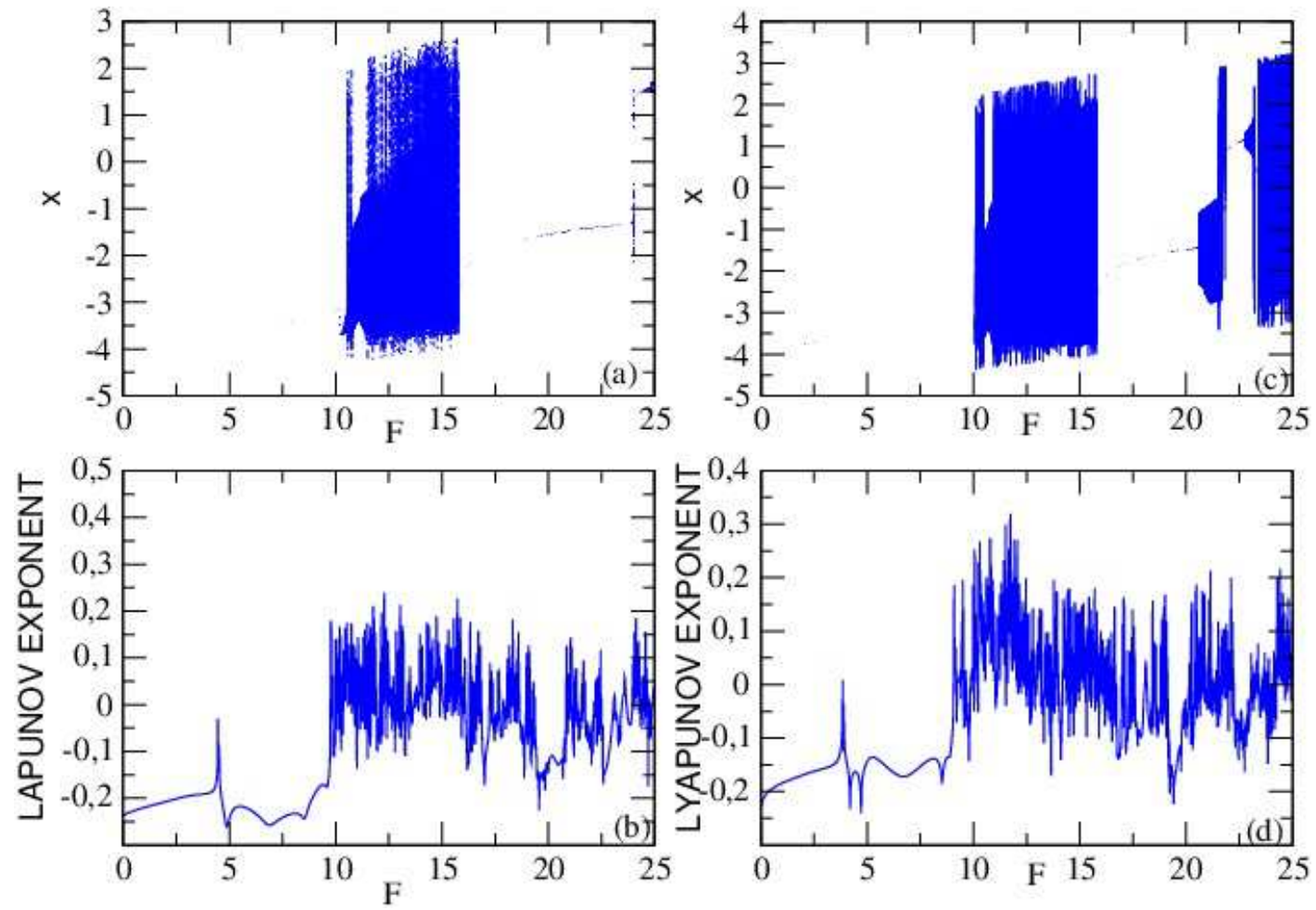

Fig. 12. Bifurcation diagram (upper frame) and Lyapunov exponent (lower frame) versus the amplitude $F$ with the parameters of Fig. 11 for $\beta=6, \alpha=0$ (left) and $\alpha=1$ (right) 

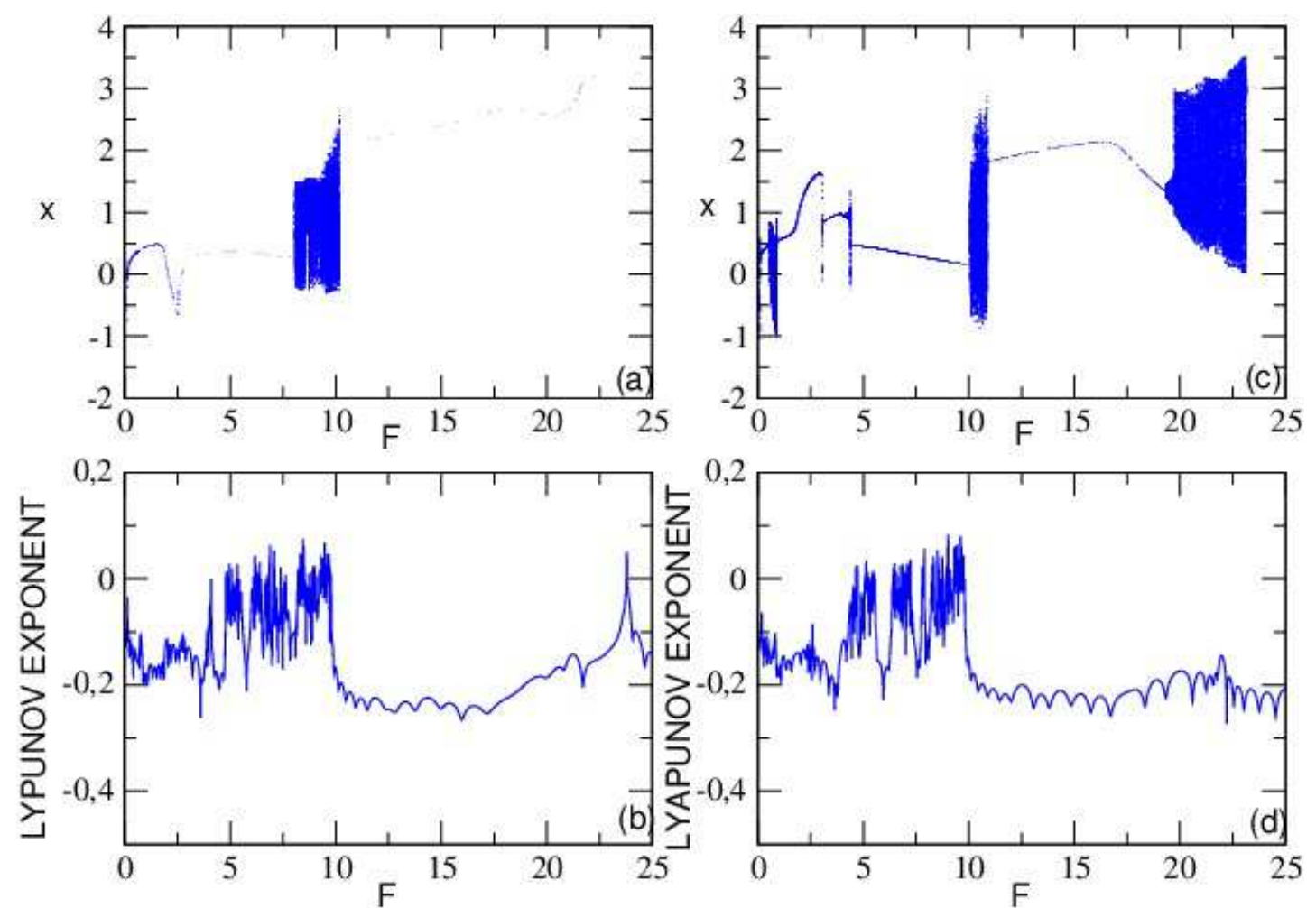

Fig. 13. Bifurcation diagram (upper frame) and Lyapunov exponent (lower frame) versus the amplitude $F$ with the parameters of Fig. 11 for $\beta=1.80 ; \epsilon=0.06, \alpha=0$ (left) and $\alpha=1$ (right)
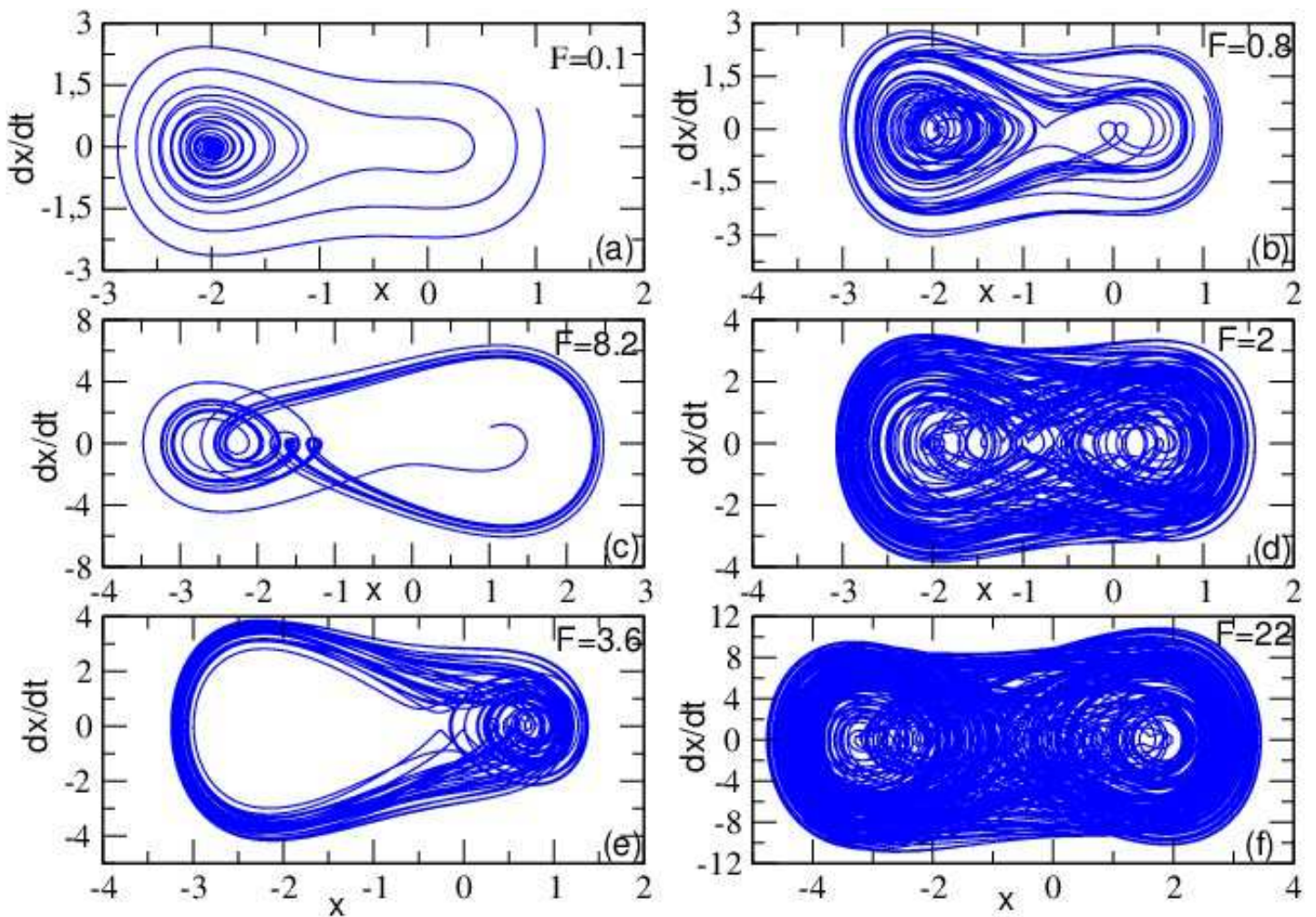

Fig. 14. Various phase portraits for several different values of $F$ with the parameters of Fig. 9 and $\alpha=0$ 

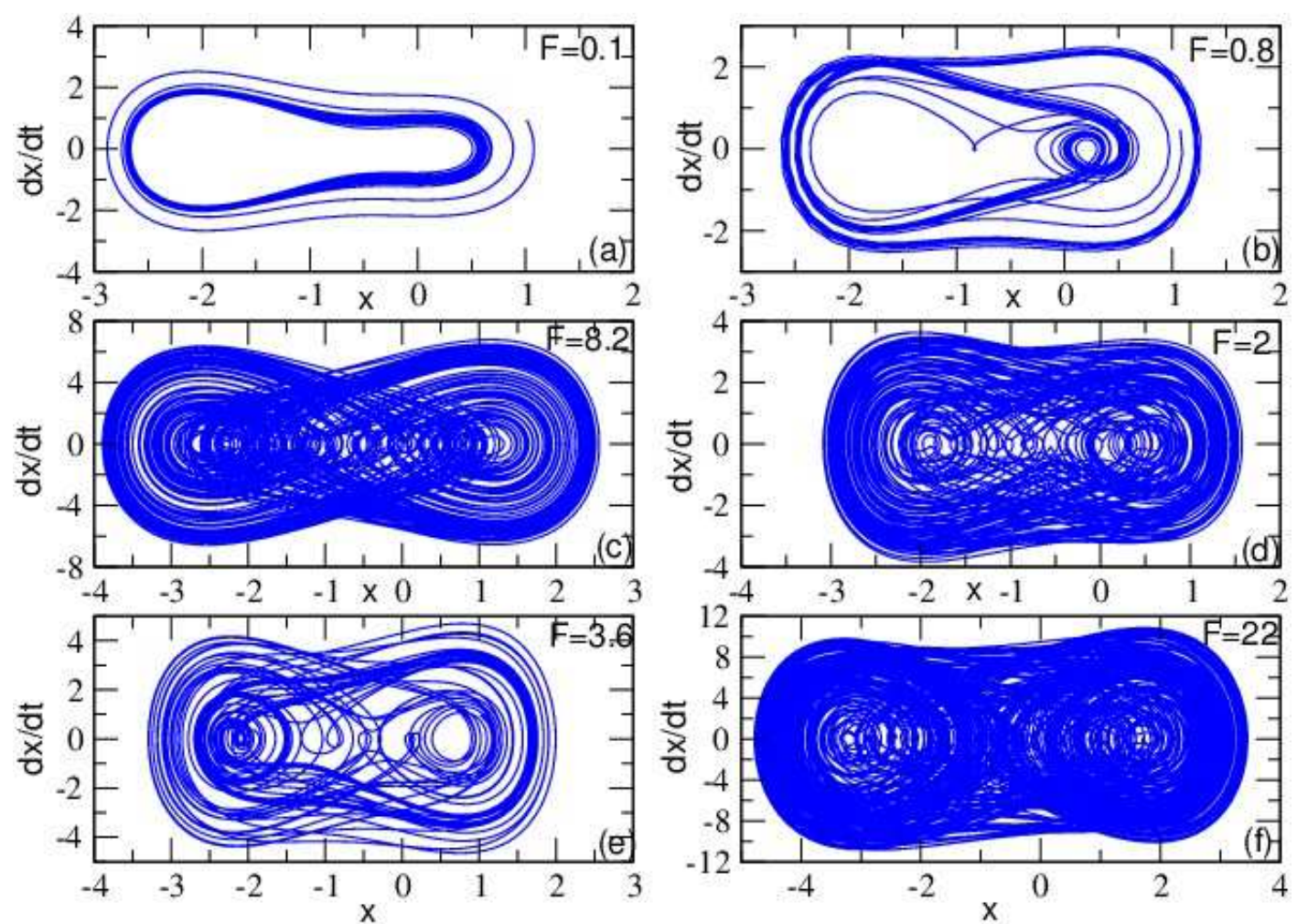

Fig. 15. Various phase portraits for several different values of $F$ with the parameters of Fig. 11 and $\alpha=1$
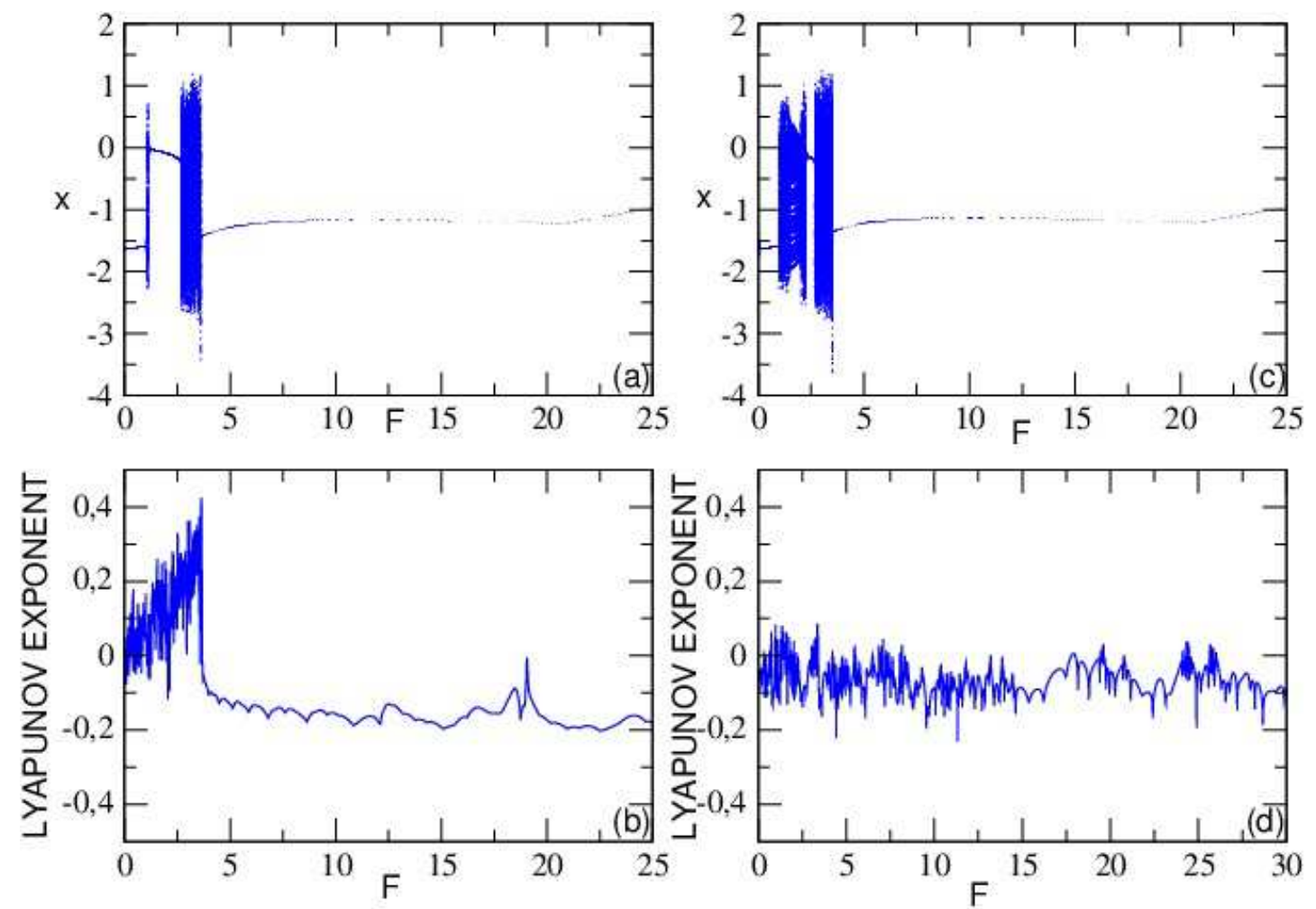

Fig. 16. Bifurcation diagram (upper frame) and Lyapunov exponent (lower frame) versus the amplitude $F$ with the parameters of Fig. 11 for $\Omega=3, \gamma=0$ (left) and $\alpha=1$ (right) 

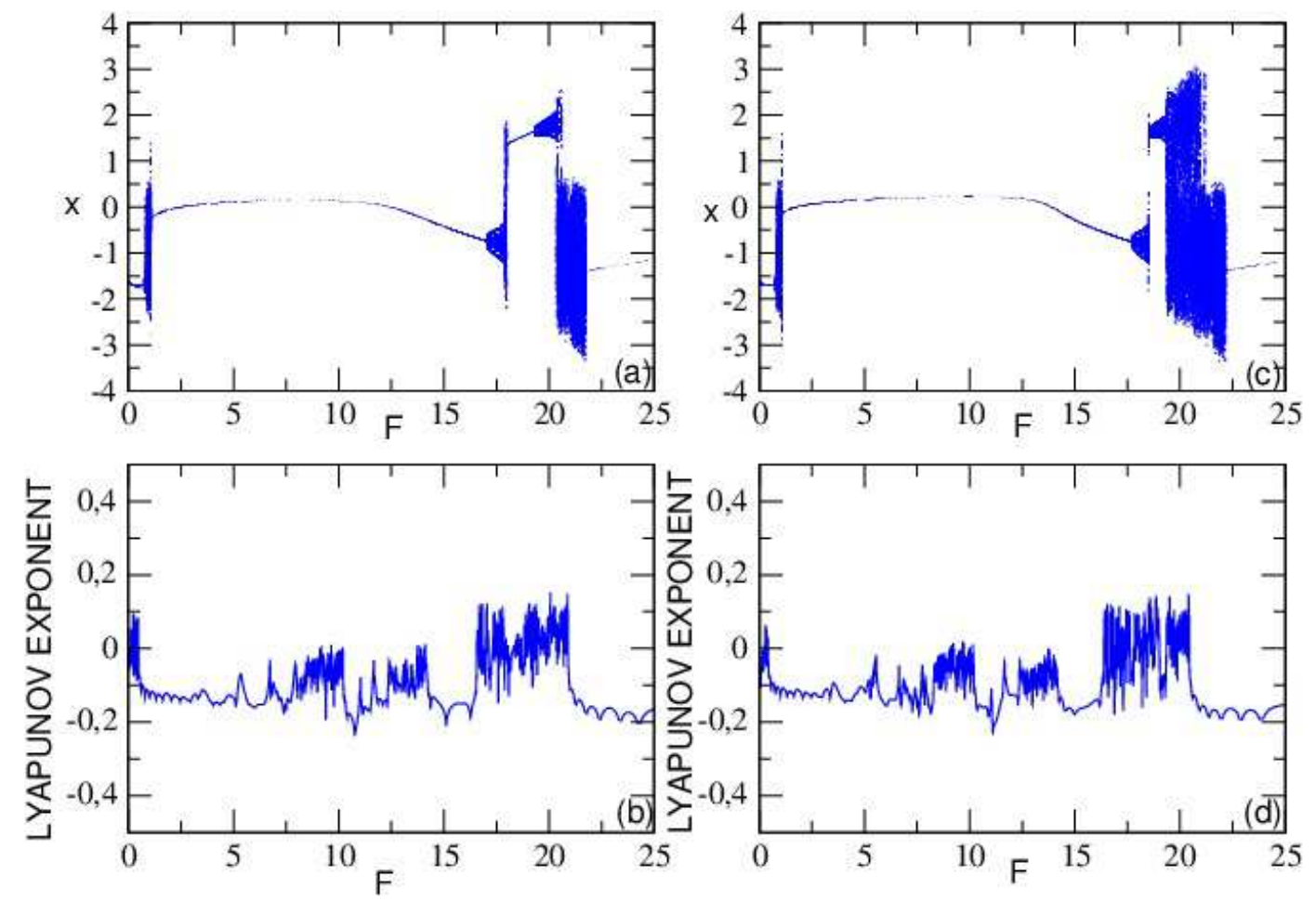

Fig. 17. Bifurcation diagram (upper frame) and Lyapunov exponent (lower frame) versus the amplitude $F$ with the parameters of Fig. 11 for $\Omega=2, \alpha=0$ (left) and $\alpha=1$ (right)
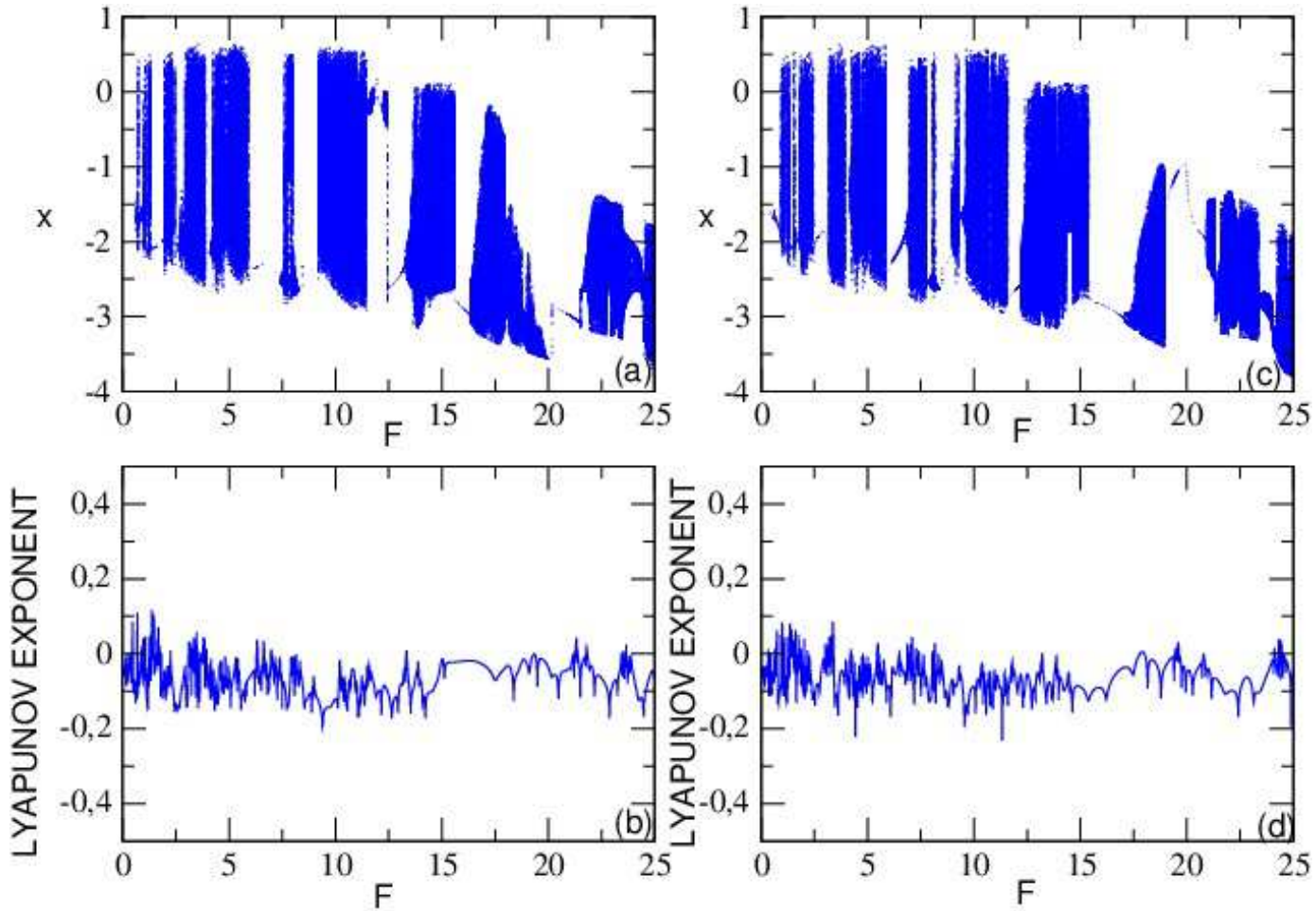

Fig. 18. Bifurcation diagram (upper frame) and Lyapunov exponent (lower frame) versus the amplitude $F$ with the parameters of Fig. 11 for $\Omega=\frac{1}{3}, \alpha=0$ (left) and $\alpha=1$ (right) 

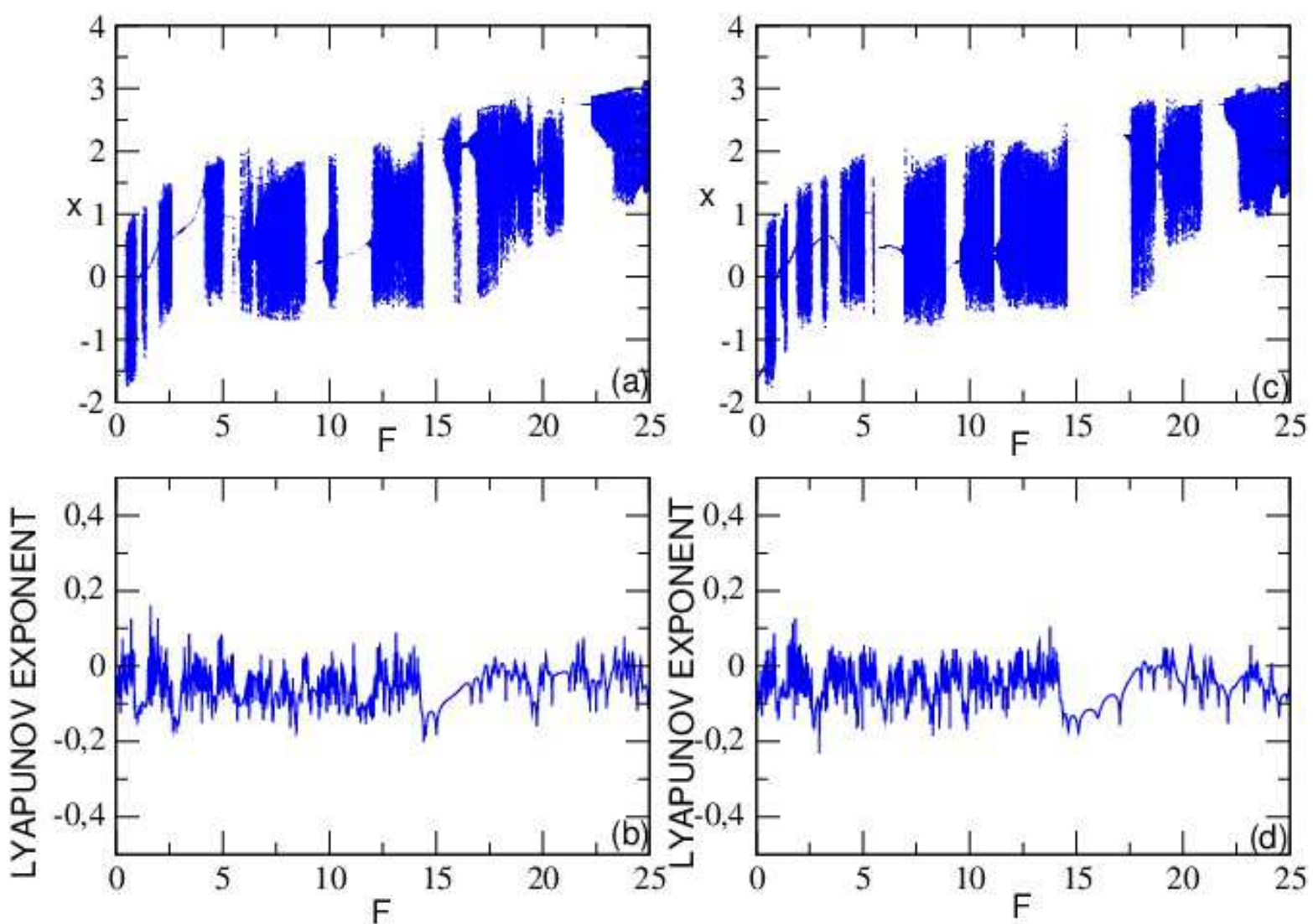

Fig. 19. Bifurcation diagram (upper frame) and Lyapunov exponent (lower frame) versus the amplitude $F$ with the parameters of Fig. 11 for $\Omega=\frac{1}{2}, \alpha=0$ (left) and $\alpha=1$ (right)

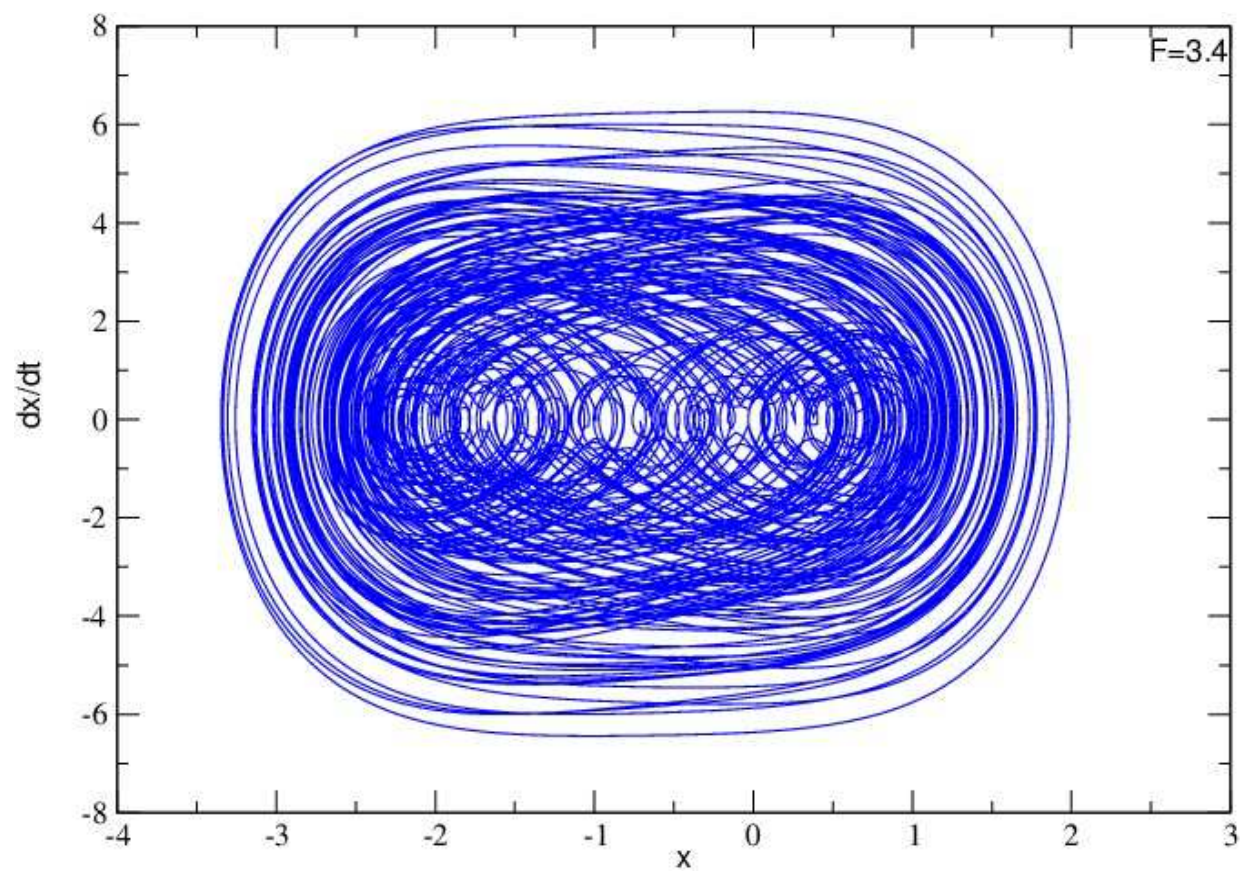

Fig. 20. Phase portrait for the first superharmonic resonance with the parameters of Fig. 16 
It should be emphasized from Fig.11 that there are some domains where the Lyapunov exponent does not match very well the regime of oscillations expected from the bifurcation diagram. Far from being an error which has occurred from the numerical simulation process, such a behavior corresponds to what is called the intermittency phenomenon. Therefore, within these intermittent domains, the dynamics of the model can't be predicted. For instance, some forecasted period-1 and quasiperiodic motions from the bifurcation diagram are not confirmed by the Lyapunov exponent. Figure 14 and 15 show the remaining of intermittency in the system according to the quadratic hybrid parameter. On the other hand, the parameters of

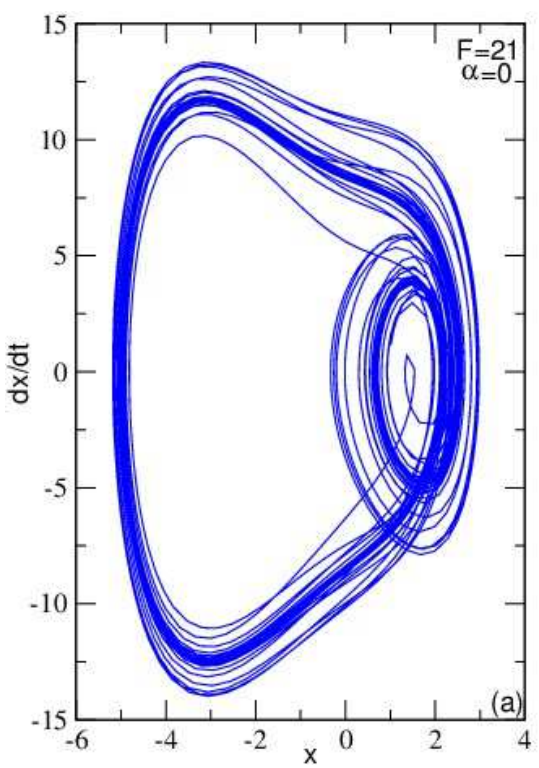

nonlinearity can influence the chaotic motion of the model. Consequently a set of physical parameters of the model, $\alpha ; \beta ; \epsilon$ and $\gamma$ and can be used to increase or dismiss the rate of chaotic motion in the model. The same simulations obtained in the subharmonic and superharmonic resonance have also been represented respectively in Fig. 16-23. Basins of chaoticity in the primary, superharmonic and subharmonic resonance are also plotted in Fig. 24-26. From these figures, we conclude that chaos is more abundant in the superhamonic and primary resonances than the subharmonic resonant states. It can be also concluded that $\alpha$ contributes to the fractalization of the basins and accentuates the chaos in the system.

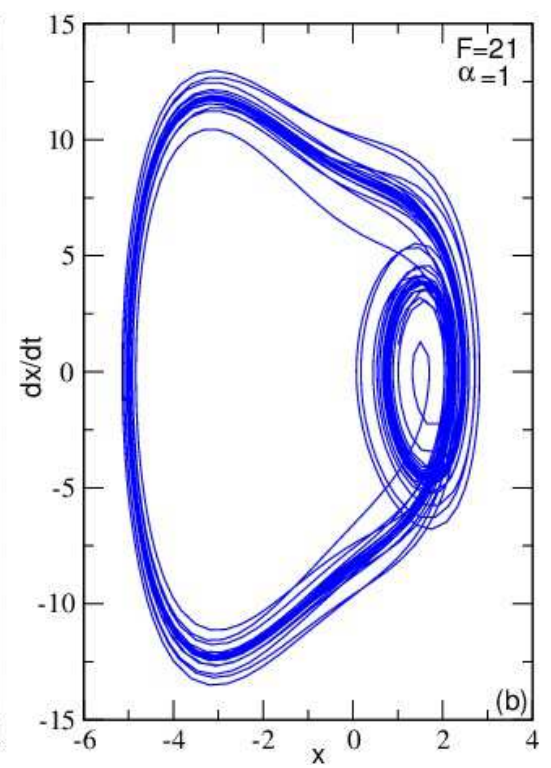

Fig. 21. Phase portrait for the second superharmonic resonance with the parameters of Fig. 17

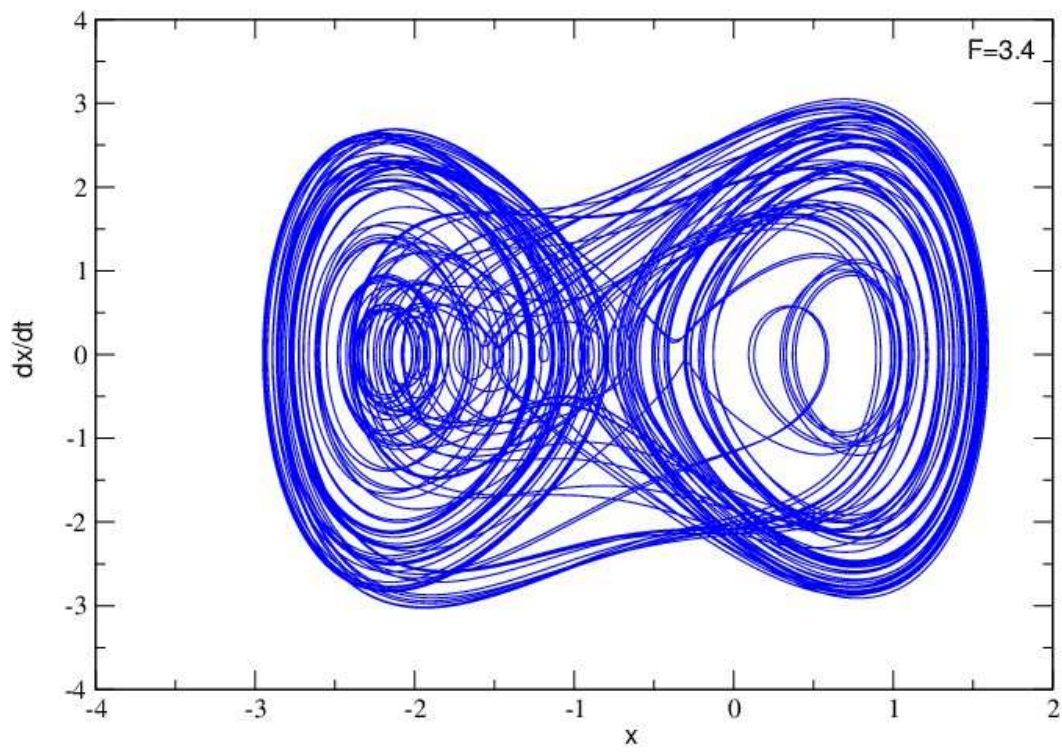

Fig. 22. Phase portrait for the first subharmonic resonance with the parameters of Fig. 18 

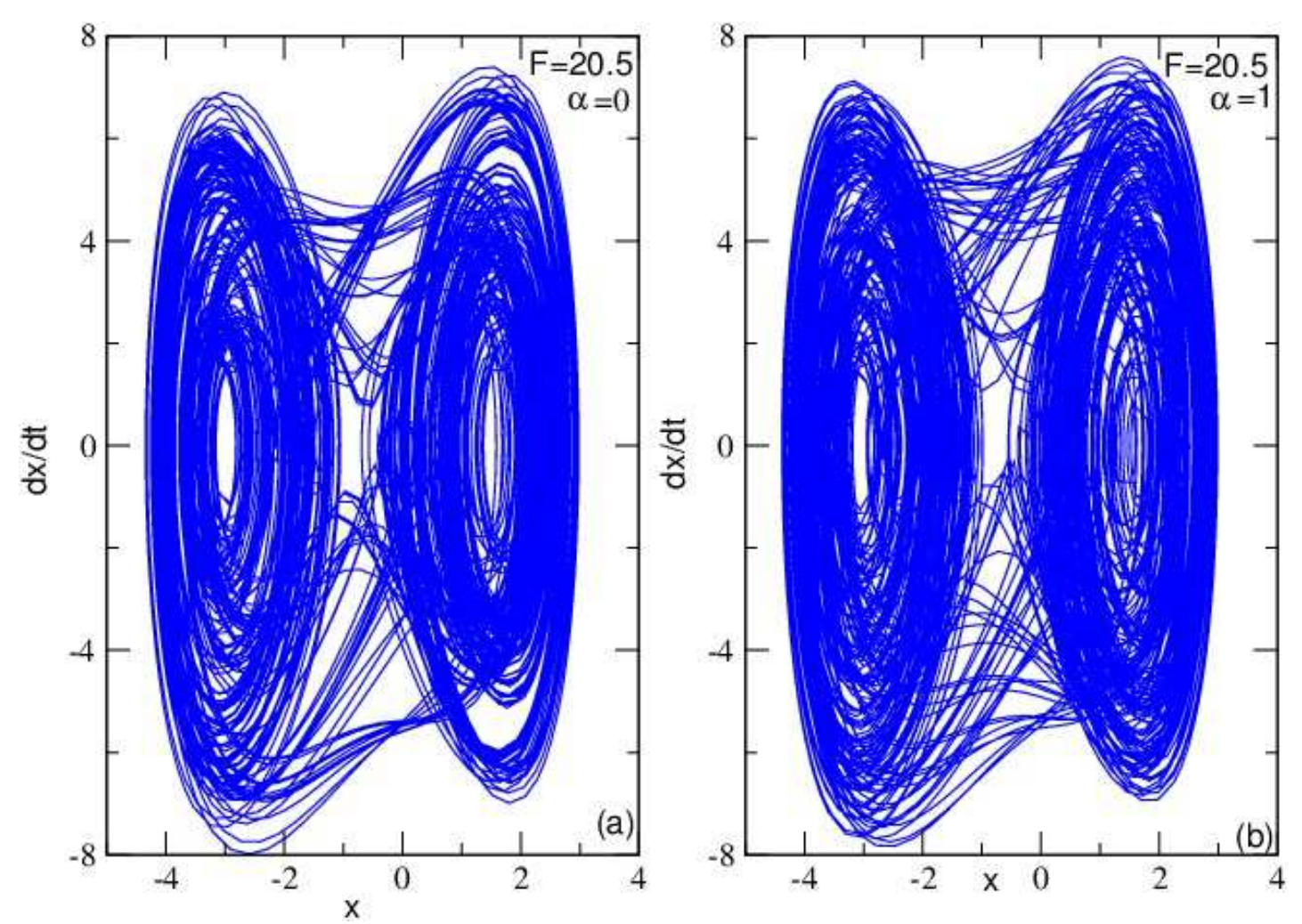

Fig. 23. Phase portrait for the second subharmonic resonance with the parameters of Fig. 19
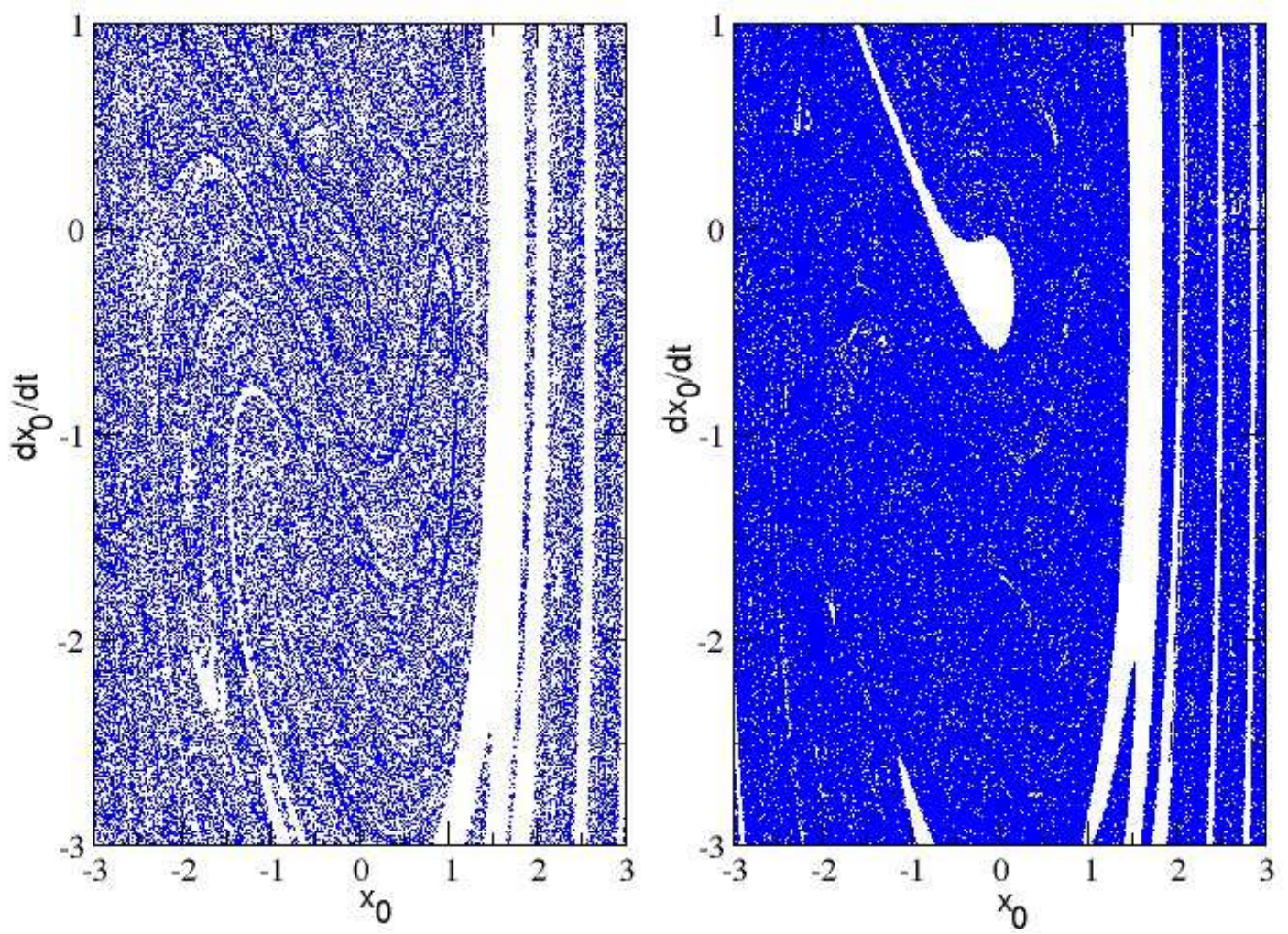

Fig. 24. Basin of chaoticity in the primary resonant state, $\alpha=0$ (left) and $\alpha=1$ (right) 

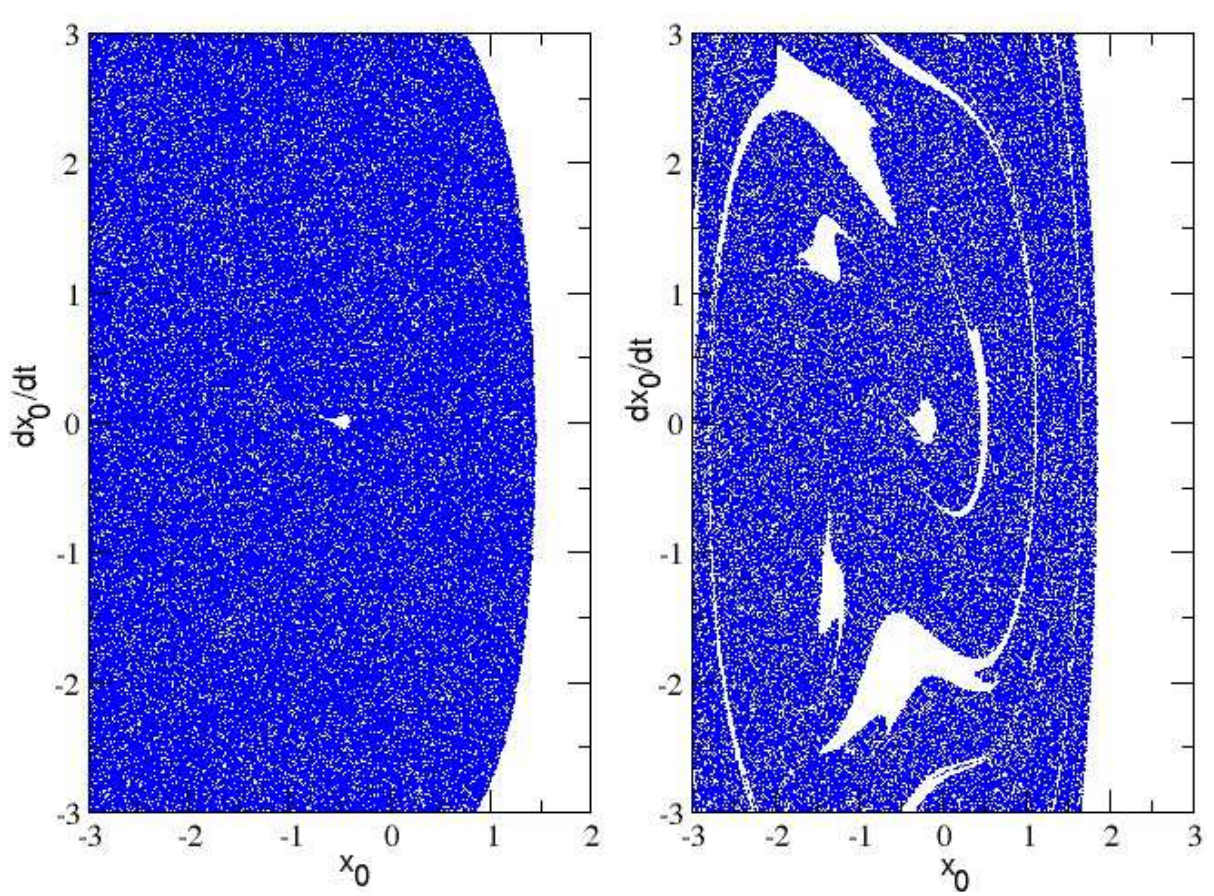

Fig. 25. Basin of chaoticity in the superharmonic resonant state, $\alpha=0$ (left) and $\alpha=1$ (right)
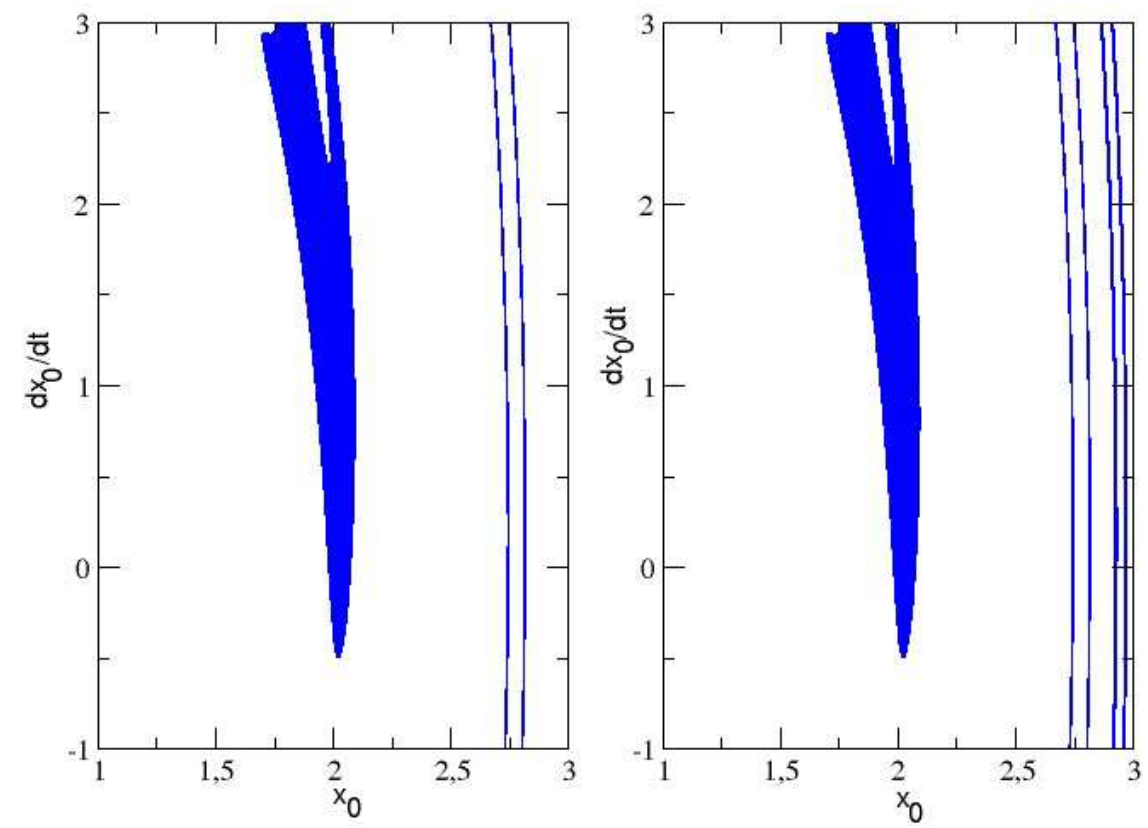

Fig. 26. Basin of chaoticity in the subharmonic resonance, $\alpha=0$ (left) and $\alpha=1$ (right).

\section{Conclusion}

In this work, we have investigated hysteresis, resonant oscillations and bifurcation mode of a system modeled by a forced modified Van der Pol-Duffng oscillator. By the multiple scales method, an exhaustive study of various resonance states is done. In the harmonic case, the harmonic balance method has enabled us to derive the amplitude of harmonic oscillations. The effects of the parameters on the behaviors of the model have been analyzed. For the resonant states case, the response amplitude stability have been derived using multiple time-scales method and perturbation method. It appears the two-order and three-order superharmonic and 
subharmonic resonances. The effects of parameters on these resonances have been found. We noticed that the hybrid and pure quadratic parameters severely influence on two-order and three-order resonances. These resonances are affected by the cubic nonlinear parameters. The influences of the dissipative parameter on the resonant, hysteresis and jump phenomena have been highlighted. Our analytical results have been confirmed by numerical simulation. Various bifurcation structures and the basin of attraction showing different types of transitions from quasi-periodic motions to periodic and chaotic motions have been drawn and the influences of different parameters on these motions have been studied. It is noticed that chaotic motions are controlled by the parameters $\epsilon, \beta, \alpha$ and $\gamma$. The results show a way to predict admissible values of the signal amplitude for a corresponding set of parameters. This could be helpful for experimentalists who are interested in trying to stabilize such a system with external forcing. Through these studies we notice that the hybrid quadratic term have not been neglected in the study of nonlinear dynamics of plasma oscillations. For practical interests, it is useful to develop tools and to find ways to control or suppress such undesirable regions. This will be also useful to control high amplitude of oscillations obtained and which are generally source of instability in plasma physics.

\section{Acknowledgment}

The authors thank very much Drs. Enjieu Kadji, Victor Kamdoum and Peguy Roussel Nwagour for their collaborations. We also thank very much the anonymous referees whose useful criticisms, comments and suggestions have helped strengthen the content and the quality of the paper.

\section{Authors Contributions}

A.V. Monwanou: Contributed in writing of the manuscript.

A.V. Monwanou, C.H. Miwadinou and L.A. Hinvi: Contributed equally to this work in the theoretical and numerical part.

J.B. Chabi Orou: Contributed to the supervision and the analysis of the results of this paper.

\section{Ethics}

The present work is not published in its present form in any journal or will not be published in any journal.

\section{References}

Ainamon, C., C.H. Miwadinou, A.V. Monwanou and J.B.C. Orou, 2014. Analysis of multiresonance and chaotic behavior of the polarization in materials modeled by a Duffing equation with multifrequency excitations. Applied Phys. Res., 6: 74-86. DOI: 10.5539/apr.v6n6p74
Arshad, M.M., T. Rafiq and G. Murtaza, 1999. Chaos in the parallel sheared plasma flow driven electromagnetic turbulence in nonuniform magnetoplasmas. Phys. Plasma, 13: 1107-1112. DOI: $10.1063 / 1.873357$

Bhattacharyya, R. and M.S. Janaki, 2006. Two-fluid stationary states with dissipation and external drive. Phys. Plasma.

Dendy, R.O., 1993. Plasma Physics: An Introductory Course. 1st Edn., Cambridge University Press, Cambridge, ISBN-10: 0521433096, pp: 533.

Enjieu, K.H.G., B.R.N. Nbendjo, J.B.C. Orou and P.K. Talla, 2008. Nonlinear dynamics of plasma oscillations modeled by an anharmonic oscillator. Phys. Plasmas, 15: 1-14. DOI: 10.1063/1.2841032

Enjieu, K.H.G., J.B.C. Orou and P. Woafo, 2007. Regular and Chaotic Behaviors of Plasma Oscillations Modeled by a Modified Duffing Equation. 1st Edn., Physica Scripta (In Press).

Hayashi, C., 1964. Nonlinear Oscillations in Physical Systems. 1st Edn., McGraw-Hill, New York, pp: 392.

Hsuan, H.C.S., 1968. Nonlinear effects in plasma resonance. Phys. Rev., 172: 137-145. DOI: 10.1103/PhysRev.172.137

Kanki, T., M. Nagata and T. Uyama, 2006. Computation of two-fluid flowing equilibrium of helicity-injected spherical torus plasma. IEEE Trans. Magnet., 42: 1403-1406. DOI: 10.1109/TMAG.2006.871391

Keen, B.E. and W.H. Fletcher, 1971. Feedback stabilization of a plasma instability by a remote modulated source at the electron cyclotron resonance frequency. Plasma Phys., 13: 419-431. DOI: $10.1088 / 0032-1028 / 13 / 5 / 009$

Keen, B.E. and W.H. Fletcher, 1972. Nonlinear plasma instability effects for subharmonic and harmonic forcing oscillations. J. Phys. A: Gen. Phys., 5: 152165. DOI: $10.1088 / 0305-4470 / 5 / 1 / 020$

Li, L.H. and M. Matsuoka, 1996. Theoretical modeling of radiation-driven plasma turbulence in an unmagnetized plasma. Radiophys. Quantum Electron., 99: 75-76.

Loverich, J. and U. Shumlak, 2006. Nonlinear full twofluid study of $m=0$ sausage instabilities in an axisymmetric $\mathrm{Z}$ pinch. Phys. Plasma, 13: 1-9. DOI: 10.1063/1.2220009

Mahaffey, R.A., 1976. Anharmonic oscillator description of plasma oscillations. Phys. Fluids, 19: 1387-1391. DOI: $10.1063 / 1.861642$

Miwadinou, C. H., Monwanou, A. V. and Chabi Orou, J. B. 2014. Active control of the parametric resonance in the modified Rayleigh-Duffing oscillator. Afr. Rev. Phys., 9: 227-235.

Miwadinou, C.H., A.L. Hinvi, A.V. Monwanou and J.B.C. Orou, 2016b. Nonlinear dynamics of a $\phi^{6}-$ modified Duffing oscillator: Resonant oscillations and transition to chaos. Nonlinear Dyn., 88: 97-113. DOI: $10.1007 / \mathrm{s} 11071-016-3232-0$. 
Miwadinou, C.H., A.V. Monwanou and J.B.C. Orou, 2015. Effect of nonlinear dissipation on the basin boundaries of a driven two-well modified RayleighDuffing oscillator. Int. J. Bifurcation Chaos, 25: 114. DOI: $10.1142 / \mathrm{S} 0218127415500248$

Miwadinou, C.H., A.V. Monwanou, A.L. Hinvi, A.A. Koukpemedji and C. Ainamon et al., 2016a. Melnikov chaos in a modified Rayleigh-Duffing oscillator with $\phi^{6}$ potential. Int. J. Bifurcation Chaos, 26: 1-13. DOI: $10.1142 / \mathrm{S} 0218127416500851$

Nayfeh, A.H., 1981. Introduction to Perturbation Techniques. 1st Edn., John Wiley and Sons, New York, ISBN-10; 0471080330, pp: 519.

Ostrikov, K. and S. Xu, 2007. Plasma-Aided Nanofabrication: From Plasma Sources to Nanoassembly. 1st Edn., John Wiley and Sons, Weinheim, ISBN-10: 3527611568, pp: 315.
Ostrikov, K., 2005. Colloquium: Reactive plasmas as a versatile nanofabrication tool. Rev. Mod. Phys., 77: 489-511. DOI: 10.1103/RevModPhys.77.489

Piskunov, N., 1980. Calcul Differentiel et Integral, Tome II. 9th Edn., MIR, Moscou.

Proud, J., 1991. Plasma Processing of Materials: Scientific Opportunities and Technological Challenges. 1st Edn., National Academy Press, Washington D.C., ISBN-10: 0309045975, pp: 75.

Rajasekar, S., S. Parthasarathy and M. Lakshmanan, 1992. Prediction of horseshoe chaos in BVP and DVP oscillators. Chaos, Solitons and Fractals, 2: 271-280. DOI: 10.1016/0960-0779(92)90036-M

Shumlak, U. and J. Loverich, 2003. Approximate Riemann solver for the two-fluid plasma model. J. Comput. Phys., 187: 620-638. DOI: 10.1016/S00219991(03)00151-7 